\title{
REVIEW ARTICLE Neutrophil chemoattractant receptors in health and disease: double-edged swords
}

\author{
Mieke Metzemaekers ${ }^{1}$, Mieke Gouwy ${ }^{1}$ and Paul Proost ${ }^{1}{ }^{1}$
}

Neutrophils are frontline cells of the innate immune system. These effector leukocytes are equipped with intriguing antimicrobial machinery and consequently display high cytotoxic potential. Accurate neutrophil recruitment is essential to combat microbes and to restore homeostasis, for inflammation modulation and resolution, wound healing and tissue repair. After fulfilling the appropriate effector functions, however, dampening neutrophil activation and infiltration is crucial to prevent damage to the host. In humans, chemoattractant molecules can be categorized into four biochemical families, i.e., chemotactic lipids, formyl peptides, complement anaphylatoxins and chemokines. They are critically involved in the tight regulation of neutrophil bone marrow storage and egress and in spatial and temporal neutrophil trafficking between organs. Chemoattractants function by activating dedicated heptahelical G protein-coupled receptors (GPCRs). In addition, emerging evidence suggests an important role for atypical chemoattractant receptors (ACKRs) that do not couple to $G$ proteins in fine-tuning neutrophil migratory and functional responses. The expression levels of chemoattractant receptors are dependent on the level of neutrophil maturation and state of activation, with a pivotal modulatory role for the (inflammatory) environment. Here, we provide an overview of chemoattractant receptors expressed by neutrophils in health and disease. Depending on the (patho)physiological context, specific chemoattractant receptors may be up- or downregulated on distinct neutrophil subsets with beneficial or detrimental consequences, thus opening new windows for the identification of disease biomarkers and potential drug targets.

Keywords: Neutrophils; chemoattractant; G protein-coupled receptors; chemokine; leukocyte migration

Cellular \& Molecular Immunology (2020) 17:433-450; https://doi.org/10.1038/s41423-020-0412-0

\section{THE NEUTROPHIL: AN INTRODUCTION}

Neutrophils are derived from a common myeloid progenitor present in the bone marrow and extramedullary tissues (e.g., spleen) and represent $50-70 \%$ of the total pool of peripheral blood leukocytes in humans (only $10-25 \%$ in mice). ${ }^{1}$ These innate leukocytes were traditionally considered simple, short-lived cells serving the rather mundane task of releasing antimicrobial agents until more specialized cells arrived at the site of inflammation to launch a more effective attack. However, the currently available, more refined technologies have indicated that neutrophils are more sophisticated cells than initially anticipated. They presumably display phenotypical and functional heterogeneity and play central roles in health and disease., ${ }^{2,3}$ Although the lifespan of neutrophils is still debated, scientific evidence supports the notion that inflammation promotes the longevity of these cells, and it is now recognized that neutrophils contribute to the initiation, modulation and resolution phases of inflammation, are involved in wound healing and are important for the maintenance of tissue homeostasis. ${ }^{3,4}$ Moreover, neutrophils can actively interact with and instruct other immune cells.

As first-line responders to infection and tissue injury, neutrophils are armed with a comprehensive set of defense mechanisms (Fig. 1). They are professional phagocytes that are able to engulf and destroy foreign material. In addition, assembly and activation of nicotinamide adenine dinucleotide phosphate (NADPH), a multiprotein electron transfer system, enables the production of reactive oxygen species (ROS), including $\mathrm{O}_{2}{ }^{-}, \mathrm{HO}$, and $\mathrm{H}_{2} \mathrm{O}_{2}$, by these cells. ${ }^{3,5}$ The latter is converted into hypochlorous acid ( $\left.\mathrm{HOCl}\right)$ by myeloperoxidase (MPO). Neutrophils carry different types of intracellular granules with specific compositions and functions. ${ }^{6}$ Azurophilic granules contain MPO, neutral proteases (elastase, cathepsin G, proteinase 3 and azurocidin) and membranepermeabilizing agents (lysozyme, defensins and bacterial permeability-increasing proteins). Consequently, these are the primary microbicidal granules. Specific and gelatinase granules show significant overlap in terms of content and function, though they can be distinguished based on the presence of lactoferrin (specific granules) and matrix metalloproteinase-9 (MMP-9; gelatinase granules). Proteins in both types of granules are vital for neutrophil extravasation and migration. Secretory vesicles provide an additional set of proteins that facilitate extravasation, such as chemoattractant receptors (e.g., formyl peptide receptor 1 (FPR1) and CXC chemokine receptor 2 (CXCR2)), CD16, Mac-1 and complement receptor 1 . Finally, the more recently described ficolin-enriched granules contain high levels of ficolin-1, a microbe-binding lectin with structural homology to the complement protein $\mathrm{C} 1 \mathrm{q}$ that, upon interaction with microorganisms, activates the complement cascade. In addition to their phagocytic capacity and potent ability to release ROS and degradative enzymes, highly activated neutrophils may release webs of

\footnotetext{
${ }^{1}$ Laboratory of Molecular Immunology, Rega Institute, KU Leuven, Herestraat 49 bus 1042, B-3000 Leuven, Belgium

Correspondence: Paul Proost (paul.proost@kuleuven.be)
}

Received: 23 December 2019 Accepted: 5 March 2020

Published online: 1 April 2020 


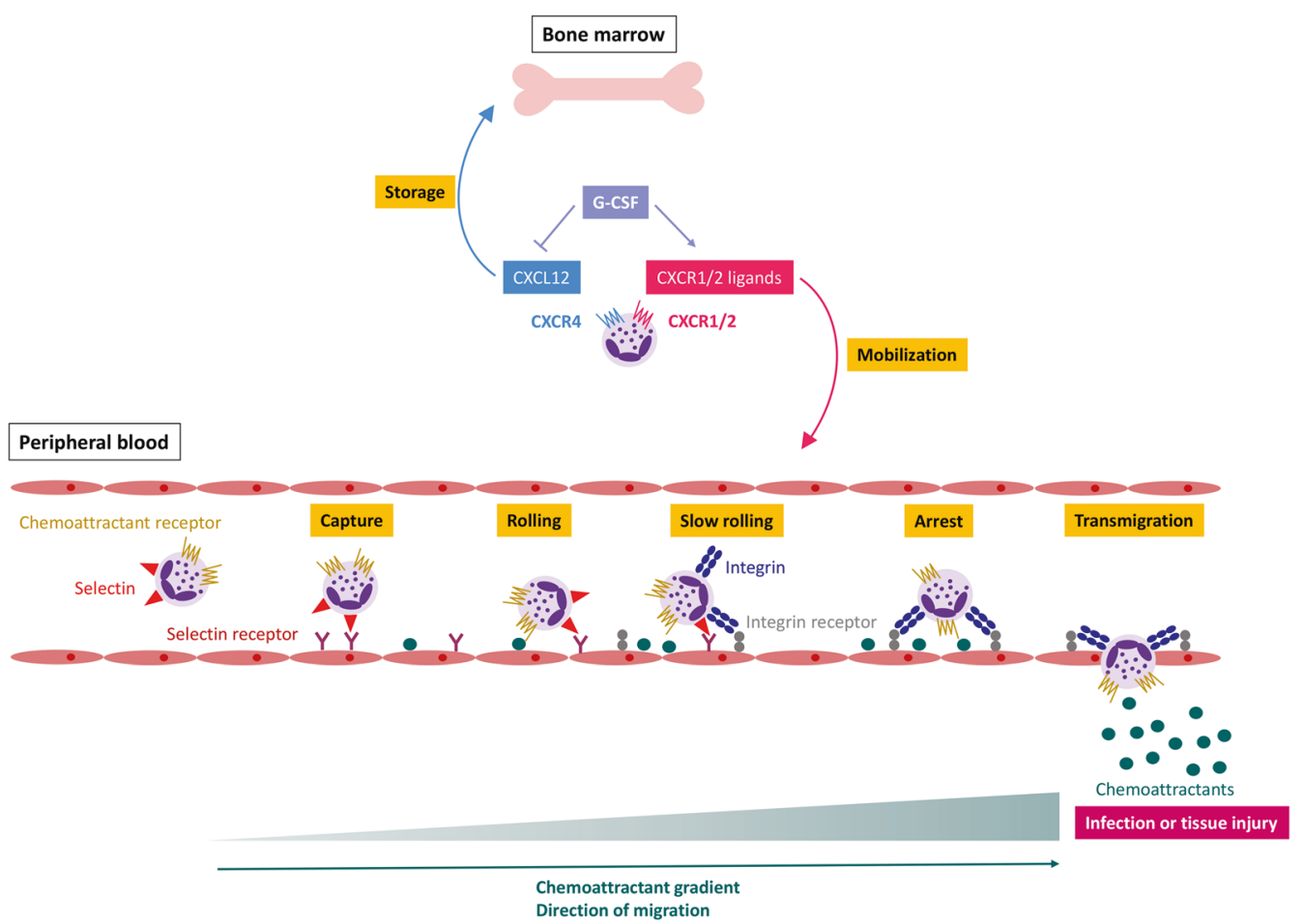

Direction of migration

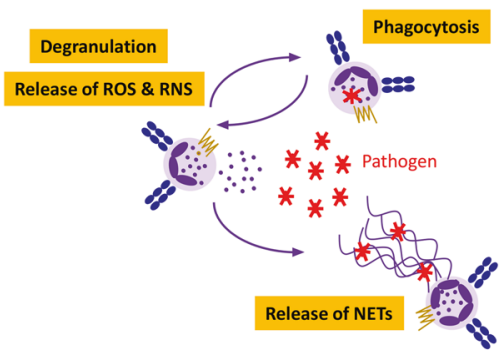

Fig. 1 Regulation of neutrophil migration and activation by chemoattractants. Inflammation or tissue injury leads to increased G-CSF production, thus stimulating neutrophil mobilization into the peripheral blood. Interactions between selectins and their receptors facilitate the initial rolling of the neutrophil along the vascular endothelium. A chemoattractant gradient determines the direction of neutrophil migration. Activation of chemoattractant receptors induces the upregulation and activation of integrins. Binding of integrins to their ligands enables tight adhesion, eventually leading to trans- or paracellular migration. At the site of infection, neutrophils can phagocytose foreign material, including pathogens, produce reactive oxygen/nitrogen species (ROS/RNS) and release degradative enzymes and microbicidal agents (via degranulation) and neutrophil extracellular traps (NETs)

extracellular DNA decorated with proteases and antimicrobial molecules, i.e., neutrophil extracellular traps (NETs). ${ }^{7}$

Self-evidently, their high cytotoxic potential implies that neutrophil activation and migration require tight regulation to prevent the exaggerated inflammation that leads to tissue damage. In vivo neutrophil trafficking and activation depend on complex collaboration between adhesion molecules, cytokines and proteases, with a central role for professional chemoattractant molecules and their cognate G protein-coupled receptors (GPCRs) (Fig. 1). Here, we provide an overview of chemoattractant receptors expressed by neutrophils and their proposed roles in health and disease.

\section{REGULATION OF NEUTROPHIL TRAFFICKING, PRIMING AND ACTIVATION BY CHEMOATTRACTANTS}

The neutrophil migration cascade comprises distinguishable phases of slow, selectin-mediated rolling activation and strengthening of adhesion that result in integrin-mediated arrest, intraluminal crawling and final transmigration (Fig. 1). ${ }^{8,9}$ Neutrophils primarily take a paracellular route, implying that they cross the vascular wall predominantly at endothelial cell junctions
(Fig. 2). Depending on the intensity of the inflammation and the vascular bed of the tissue involved, they may also follow a transcellular path and migrate directly though the endothelial cell body without loss of the integrity of the plasma membrane of either cell. Multiple steps of the neutrophil migration cascade depend on chemoattractants and their receptors. These molecular guidance cues enable concentration-dependent neutrophil migration, which determines the direction of intravascular crawling, triggers adhesion strengthening and is involved in the crucial final step of transmigration (vide infra). ${ }^{10,11}$ In humans, neutrophil chemoattractants belong to four biochemically distinct subfamilies, i.e., chemotactic lipids (e.g., leukotriene $B_{4}$ or $\mathrm{LTB}_{4}$ ), chemokines (CXCL1 to CXCL3 and CXCL5 to CXCL8 in humans), complement anaphylatoxins ( $\mathrm{C} 3 \mathrm{a}$ and $\mathrm{C} 5 \mathrm{a}$ ) and formyl peptides (e.g., N-formyl-Met-Leu-Phe or fMLF). They all function by activating dedicated GPCRs with seven membrane-spanning helices.

At first glance, the similarities in chemoattractant functions and the features of their cognate receptors may give the impression that these molecules are highly redundant. Nevertheless, convincing scientific evidence shows that each chemoattractant family contributes to neutrophil trafficking in a unique manner (Fig. 2). 
A
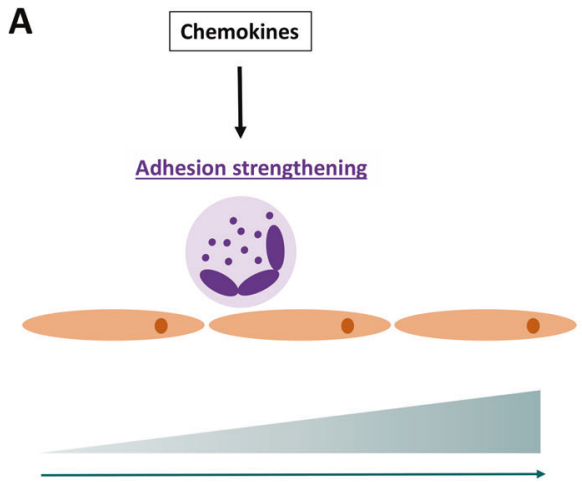

Concentration-dependent, directional migration

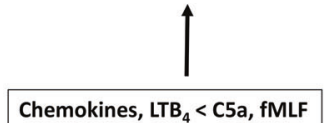

B

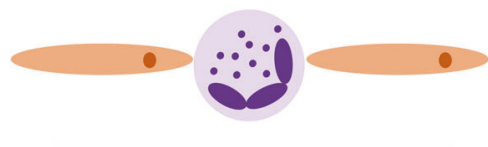

Breaching of endothelial cell junctions, paracellular extravasation

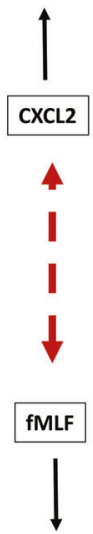

Transcellular extravasation

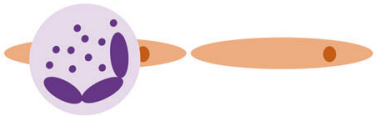

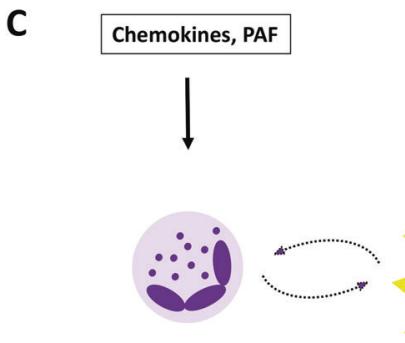

Naive neutrophil

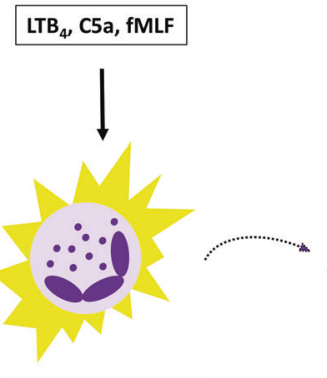

Primed neutrophil

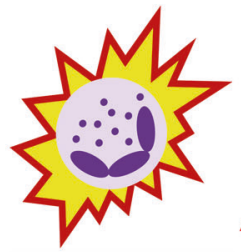

Activated neutrophil

Fig. 2 Examples of potential chemoattractant complementarity and collaboration. Different chemoattractant families and their receptors may fulfill complementary roles during neutrophil migration and activation in vivo. a Migrating neutrophils can discriminate between intermediate-target (chemokines and $\mathrm{LTB}_{4}$ ) and end-target (C5a and fMLF) chemoattractants and will eventually follow end-target signals. $\mathbf{b}$ Locally produced chemoattractants may preferentially stimulate neutrophil transmigration via the trans- or paracellular route. c Chemokines and PAF act as priming agents that push the neutrophil towards a state of 'high alert', resulting in a more aggressive response upon subsequent exposure to activating signals such as those from C $5 \mathrm{a}$, fMLF and $\mathrm{LTB}_{4}$

First, differences in cellular sources and temporal and spatial production may partially explain the nonredundant roles of chemoattractants in vivo. Second, intermediate-target ( $\mathrm{LTB}_{4}$ and chemokines) can be distinguished from end-target chemoattractants (formyl peptides and C5a). Indeed, in a complex (patho)physiological environment, neutrophils can prioritize chemoattractants to eventually follow the signals transmitted by the end-target molecules. ${ }^{10,11}$ Thus, chemoattractants are believed to cooperate spatiotemporally, in a hierarchical manner, to orchestrate neutrophil migration. Impressive previous research efforts have illustrated the in vivo relevance of this hypothesis. For instance, analysis of sterile inflammation in mice by intravital imaging revealed that initial neutrophil chemotaxis in healthy tissue directed towards the site of inflammation depends on an intravascular chemokine gradient, whereas the final migration step into the injured tissue is governed by formyl peptides derived from dying cells. ${ }^{12}$ Similarly, CXCR2-interacting chemokines and FPR-activating mitochondrial products were found to act in concert to drive neutrophil migration and full-blown inflammation in a murine model of acetaminophen-induced liver cytotoxicity. ${ }^{13}$
Importantly, members of distinct chemoattractant families presumably serve different functions during the final step of neutrophil transmigration. For instance, the chemokine CXCL2, partially released by neutrophils, fulfills a determinative role in the adequate breaching of endothelial cell junctions to facilitate paracellular migration (Fig. 2b). ${ }^{14}$ Conversely, the bacterial-derived chemoattractant fMLF drives neutrophil extravasation via the transcellular pathway (Fig. 2b). ${ }^{9}$

The descriptor 'chemoattractant' may insinuate that the function of these molecules is restricted to leukocyte migration. However, chemoattractants and their cognate receptors are also implicated in neutrophil activation, a highly dynamic process designed to guarantee that maximal activation manifests at the correct location in the body and only when necessary. ${ }^{15}$ Circulating neutrophils are quiescent under homeostatic conditions but may enter a state of 'high alert' upon confrontation with inflammatory agents-including chemokines and plateletactivating factor (PAF)—or exposure to mechanical stress (Fig. 2c). The primed phenotype may be transient or enduring, depending on the priming agent and context. Primed neutrophils may 
436

become fully activated or can undergo a spontaneous, nearcomplete reversal into the quiescent phenotype, highlighting the apparent plasticity of these leukocytes. Compared to the action of quiescent cells, primed neutrophils respond more aggressively upon successive stimulation by activating signals such as those transduced by $\mathrm{LTB}_{4}, \mathrm{fMLP}$ and $\mathrm{C} 5 \mathrm{a}$, as illustrated by enhanced ROS production, degranulation, phagocytosis and an increased tendency to release NETs.

\section{Chemotactic lipids}

$\mathrm{LTB}_{4}$ elicits chemotactic responses by a variety of leukocytes but is likely best known as a potent, intermediate-target neutrophil chemoattractant. During inflammation, $\mathrm{LTB}_{4}$ is rapidly produced by de novo synthesis in response to inflammatory cytokines (e.g., interleukin-1 (IL-1) and tumor necrosis factor-a (TNF-a)), bacterial products, such as lipopolysaccharide (LPS), or other chemoattractants (PAF and $\mathrm{C} 5 \mathrm{a}$ ). ${ }^{16} \mathrm{LTB}_{4}$ is predominantly released by mature myeloid cells, with neutrophils serving as important producers of this arachidonic acid metabolite. ${ }^{17}$ Phospholipase $A 2\left(\mathrm{PLA}_{2}\right)$ is critically involved during the initial steps of the $\mathrm{LTB}_{4}$ biosynthesis cascade. ${ }^{16}$ This enzyme facilitates the mobilization of arachidonic acid to the nuclear membrane. Sequestration of arachidonic acid by the 5-lipoxygenase (5-LO)-activating protein (FLAP) eventually leads to physiological changes in the nuclear membrane, thereby facilitating recruitment of 5-LO. The latter converts arachidonic acid into 5-hydroperoxyeicosatetraenoic acid (5-HPETE), which is metabolized into LTA . The addition of a water molecule by $\mathrm{LTA}_{4}$ hydrolase ultimately leads to the generation of $\mathrm{LTB}_{4}$.

A second chemotactic lipid, PAF, has pleiotropic functions, including the regulation of leukocyte migration and activation. Strictly speaking, the term 'PAF' refers to a heterogeneous class of molecules that can be distinguished based on the saturated or mono-/di-unsaturated alkyl, acyl, or alkenyl chains present at the sn-1 position of the glycerol backbone. ${ }^{18}$ Among the different PAF species, molecules containing C16:0, C18:0, or C18:1 alkyl groups have the greatest biological potency. Cytokines such as IL-1, IL-6, IL-12 and TNF- $a$ and the hormones angiotensin II and endothelin, among others, induce PAF production in platelets and a variety of (immune) cells. PAF can be generated by structural modification of distinct membrane phospholipids (remodeling pathway). Alternatively, PAF may be synthesized de novo via a multistep enzymatic process, starting with elementary molecules such as dihydroxyacetone phosphate.

\section{Complement anaphylatoxins}

Complement precursor proteins are synthesized by the liver and circulate in peripheral blood as inactive molecules. Three modes of complement activation, i.e., the classical, alternative or lectinmediated pathway, eventually facilitate the proteolytic splicing of the fifth complement component (C5) to generate the anaphylatoxin $\mathrm{C} 5 \mathrm{a}$ and $\mathrm{C} 5 \mathrm{~b}$. $\mathrm{C} 5 \mathrm{~b}$ is the first protein to be activated in the membrane-attack complex. C5a, undoubtedly, is the predominant complement activation product with chemotactic properties and end-target signals for neutrophil recruitment. $^{19}$ Carboxypeptidases remove the $\mathrm{COOH}$-terminal Arg residue from $\mathrm{C} 5 \mathrm{a}$, generating a des-Arg form with strongly impaired biological potency. In addition to $\mathrm{C} 5 \mathrm{a}$, the complement C3-derived protein $\mathrm{C} 3 \mathrm{a}$ also has chemotactic activity. However, although its receptor has been detected on neutrophils, C3a presumably exhibits no direct neutrophil-chemotactic activity (vide infra).

\section{Formyl peptides}

Peptides composing a formyl Met (fMet) in their $\mathrm{NH}_{2}$-terminal sequence are naturally released upon degradation of bacterial components or mitochondrial products and form pathogenassociated and damage/danger-associated molecular patterns (PAMPs and DAMPs), respectively, recognized primarily by leukocytes of innate immunity. ${ }^{20-22}$ The prototype of a fMetcontaining peptide, $\mathrm{fMLF}$, is a bacterial product that targets various cell types and acts as an end-target chemoattractant during neutrophil migration.

\section{Chemokines}

Chemokines are specialized chemotactic cytokines produced by a variety of cells, including leukocytes and connective tissue cells. ${ }^{23}$ Almost 50 chemokine ligands and at least 19 cognate GPCRs have been identified. They may fulfill homeostatic and/or inflammatory functions and can be produced constitutively or in response to microbial or endogenous inflammatory mediators, depending on the ligand considered. Based on the number and spacing of the conserved $\mathrm{Cys}$ residues present in the $\mathrm{NH}_{2}$-terminal region, a classification of $\mathrm{CC}, \mathrm{CXC}, \mathrm{CX}_{3} \mathrm{C}$ and $\mathrm{C}$ chemokines is respected. Importantly, members of the chemokine family differ from all other chemoattractants by showing a certain degree of selectivity for specific leukocyte subsets. However, inflammation may alter the expression pattern of chemokine receptors on leukocytes to expand the target cell repertoire of distinct chemokines (vide infra).

In humans, seven CXC chemokines (i.e., CXCL1 to CXCL3 and CXCL5 to CXCL8) contain a conserved Glu-Arg-Leu (ELR) motif preceding the first $\mathrm{NH}_{2}$-terminal $\mathrm{Cys}$ and are considered intermediate-target signals for neutrophil chemotaxis, with CXCL8 being the prototype and most potent neutrophil-attracting and neutrophil-activating human chemokine. ${ }^{24}$ In addition, CXCL12 is critically involved in the regulation of neutrophil bone marrow storage and release (vide infra and Fig. 1). Although chemokines primarily function by activating designated GPCRs, their precise activity and availability depend on a set of multidimensional regulatory mechanisms, which may, at least partially, explain their nonredundant roles in vivo. ${ }^{25}$ In particular, it has been established that alternative gene splicing, modulation of gene transcription and mRNA stability, mutual synergism/antagonism, binding to glycosaminoglycans, interactions with atypical chemokine receptors (ACKRs) and posttranslational modifications of chemokines and GPCRs all play important roles in controlling in vivo chemokine function. ${ }^{25,26}$ Chemokines are susceptible to posttranslational truncation, citrullination, nitration and glycosylation, with ligand- and modification-dependent consequences for their biological functions. ${ }^{27}$

\section{GENERAL ASPECTS OF CHEMOATTRACTANT-INDUCED RECEPTOR SIGNALING}

Chemoattractant receptors are rhodopsin-like class A GPCRs that transduce signals via heterotrimeric $G$ proteins. They contain seven transmembrane domains interconnected by three intracellular and three extracellular loops. The $\mathrm{NH}_{2}$ and $\mathrm{COOH}$-terminal receptor domains are situated extracellularly and intracellularly, respectively. Most cellular responses elicited upon ligand-induced activation of chemoattractant receptors are sensitive to pertussis toxin, implying that these receptors predominantly couple to the inhibitory type of $\mathrm{Ga}$ protein $\left(\mathrm{Ga}_{\mathrm{i}}\right)$. Platelet-activating factor receptor (PAFR) can also couple to $\mathrm{Ga}_{\mathrm{q}}$; however, PAFR-initiated chemotaxis truly depends on $\mathrm{Ga}_{\mathrm{i}}$-mediated signaling events. ${ }^{28}$ Upon agonist stimulation, chemoattractant receptors undergo a conformational change to facilitate the exchange of guanosine diphosphate (GDP) for guanosine trisphosphate (GTP) and dissociation of the $\mathrm{Ga}_{\mathrm{i}}$ subunit from the $\mathrm{G} \beta \gamma$ dimer (Fig. 3). ${ }^{29} \mathrm{Ga}_{\mathrm{i}}$ triggers the inhibition of adenylyl cyclase (AC), consequently lowering the endogenous concentrations of the second messenger, cyclic adenosine monophosphate (cAMP). The G $\beta Y$ dimer most likely initiates at least two downstream signaling cascades in parallel. Activation of the membrane-bound enzyme phospholipase $C(P L C) \beta$, on the one hand, results in the conversion of phosphatidylinositol $(4,5)$-bisphosphate $\left(\mathrm{PIP}_{2}\right)$ into diacylglycerol 


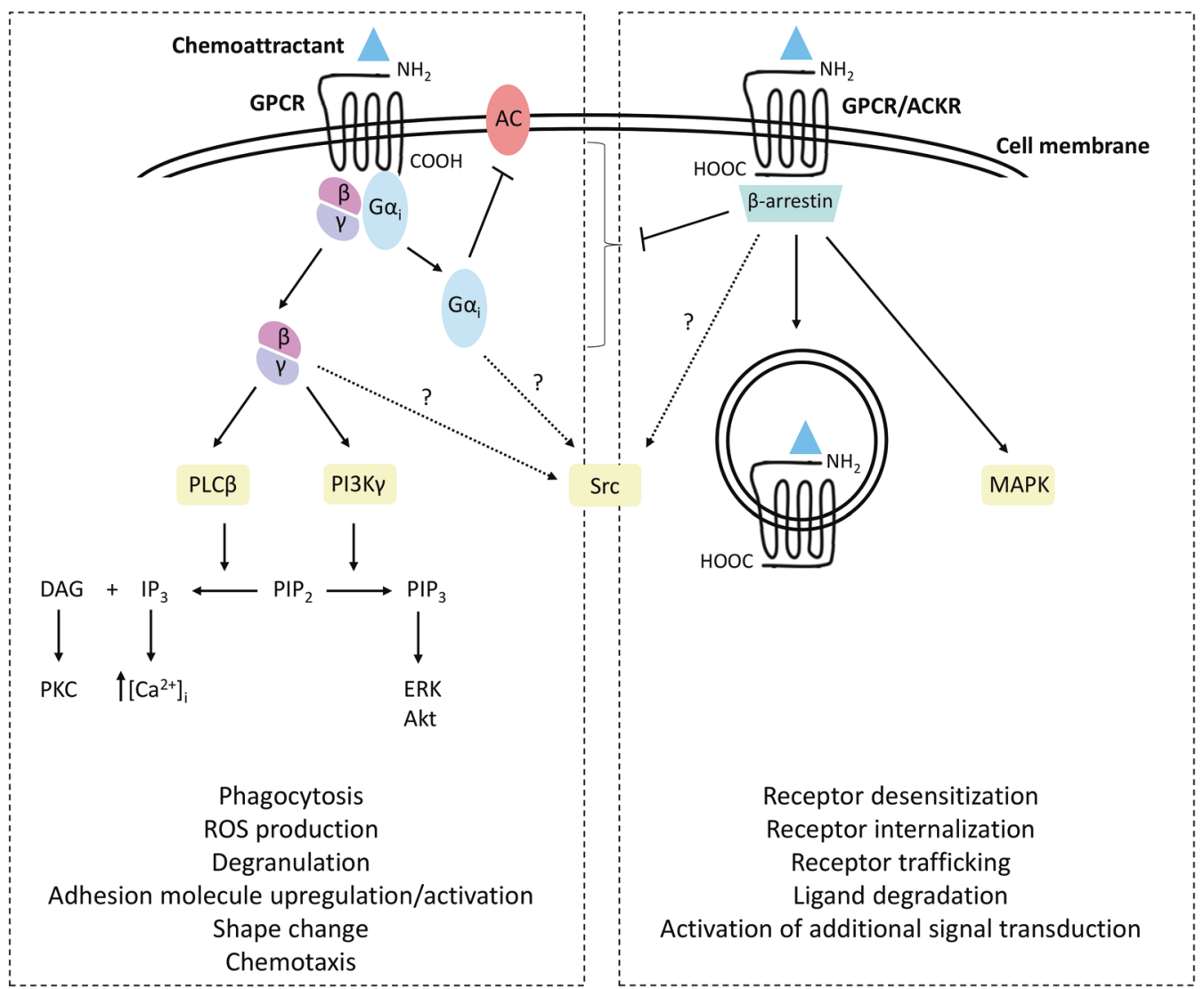

Fig. 3 Downstream effects of chemoattractant-induced GPCR activation. Activation of chemoattractant receptors by their cognate ligands evokes a conformational change and the exchange of guanosine diphosphate (GDP) for guanosine trisphosphate (GTP), followed by dissociation of the heterotrimeric $\mathrm{G}$ protein into a $\mathrm{G} \alpha_{\mathrm{i}}$ subunit and a $\mathrm{G} \beta \gamma$ dimer. The $\mathrm{G} \alpha_{\mathrm{i}}$ subunit mediates the inhibition of adenylyl cyclase (AC), resulting in a reduction in intracellular cyclic adenosine monophosphate (cAMP) concentrations. The G $\beta \gamma$ dimer initiates two parallel signaling cascades. Activation of phospholipase $C \beta(P L C \beta)$ enables the processing of phosphatidylinositol $(4,5)$-bisphosphate $\left(P I P_{2}\right)$ into diacylglycerol (DAG) and inositol 1,4,5-trisphosphate $\left(\mathrm{IP}_{3}\right)$ and the downstream activation of protein kinase $\mathrm{C}(\mathrm{PKC})$ isoforms. Second, the activation of phosphoinositide 3-kinase $\gamma\left(\mathrm{PI} 3 \mathrm{~K} \gamma\right.$ ) facilitates the conversion of $\mathrm{PIP}_{2}$ into phosphatidylinositol $(3,4,5)$-trisphosphate $\left(\mathrm{PIP}_{3}\right)$ and the downstream activation of extracellular signal-regulated kinases (ERK) and phosphokinase B (Akt). Activation of Src kinases presumably occurs via an independent yet uncharacterized pathway (indicated by question marks). Phosphorylation of the receptor at the $\mathrm{COOH}$-terminus enables binding of the $\beta$-arrestin adaptor proteins that uncouple the receptor during conventional $\mathrm{G}$ protein-dependent intracellular events

(DAG) and inositol 1,4,5-trisphosphate (IP 3$)$, ultimately leading to the mobilization of intracellular $\mathrm{Ca}^{2+}$ from the endoplasmic reticulum and the activation of $\mathrm{Ca}^{2+}$-sensitive protein kinases, including protein kinase $C$ (PKC) isoforms. On the other hand, a second enzyme is activated by the $\mathrm{G} \beta \gamma$ dimer, i.e., phosphoinositide 3-kinase $(\mathrm{PI} 3 \mathrm{~K}) \gamma$, which induces the conversion of $\mathrm{PIP}_{2}$ into phosphatidylinositol $(3,4,5)$-trisphosphate $\left(\mathrm{PIP}_{3}\right)$ and activation of extracellular signal-regulated kinases (ERK) and phosphokinase $B$ (PKB; also known as Akt) (Fig. 3).

Neutrophils express three Src tyrosine kinases, Hck, Fgr and Lyn, which may also be implicated in chemoattractant-induced signaling. ${ }^{30}$ Functionally, Src kinase activation seems to be important for the downstream activation of p38 mitogenassociated protein kinases (MAPKs). ${ }^{31}$ The mechanism of Src tyrosine kinase activation in this context has only been partially deciphered but may involve $\beta$-arrestins (vide infra), $G$ protein subunits and/or the GPCR itself and presumably manifests in parallel with the activation of PI3K-dependent and PLC $\beta$ dependent signaling (Fig. 3). ${ }^{29}$ Importantly, intermediate-target and end-target chemoattractants differ in terms of their downstream signaling pathways. Specifically, receptor activation induced by intermediate-target chemoattractants depends on PI3K- and phosphatase and tensin homolog (PTEN)-dependent pathways, while end-target signals induce Src kinase-dependent p38 MAPK phosphorylation. ${ }^{10,11}$ Nota bene, it was suggested that the end-target receptors exhibit a higher constitutive activity than the intermediate-target receptors. ${ }^{32}$

A hallmark feature of GPCRs, including those serving as chemoattractant receptors, is the typical rapid homologous desensitization of the receptor upon agonist stimulation. Homologous desensitization is predominantly accomplished through the phosphorylation of the activated receptor by intracellular GPCR kinases (GRKs) and consecutive docking of $\beta$-arrestin adaptor proteins, eventually leading to $G$ protein uncoupling. ${ }^{33}$ Importantly, independent of their ability to uncouple GPCRs from conventional signaling, $\beta$-arrestins may directly activate downstream pathways to fine-tune many migration-related intracellular events. ${ }^{33,34}$ Additionally, interactions between chemokines and ACKRs-which do not couple to $G$ proteins-usually result in $\beta$-arrestin-dependent signaling responses (Fig. 3). Furthermore, different chemoattractant families and their cognate receptors may undergo heterologous desensitization; for example, reciprocal desensitization of fMLFand $\mathrm{C} 5 \mathrm{a}$-induced $\mathrm{Ca}^{2+}$ signaling, actin polymerization and migration of human neutrophils has been reported. ${ }^{35,36}$ Moreover, prestimulation with either of these chemoattractants induces a unidirectional desensitization of CXCL1 and CXCL8, thus inhibiting the neutrophil responses they elicit. In mice, stimulation by a nonpeptidyl FPR agonist results in the downregulation of CXCR2 expression on neutrophils and failure 
438

to respond to $\mathrm{C} 5 \mathrm{a}, \mathrm{LTB}_{4}$, or CXCL1 in calcium mobilization and chemotaxis assays in vitro. ${ }^{37}$ In contrast, PAFR activation facilitates the reactivation of desensitized FPRs on neutrophils in a unidirectional, actin-dependent manner, resulting in enhanced superoxide production. ${ }^{38}$ It was recently demonstrated that PAFR-initiated $\mathrm{Ga}_{\mathrm{q}}$-related signals are critical for the activation of $\mathrm{Ga}_{\mathrm{i}}$-coupled FPRs. ${ }^{39}$

\section{EXPRESSION LEVELS AND FUNCTIONS OF NEUTROPHIL CHEMOATTRACTANT RECEPTORS IN HEALTH AND DISEASE}

Receptors for chemotactic lipids

BLT1. BLT1 is the high-affinity receptor for the prototype chemotactic lipid $\mathrm{LTB}_{4}{ }^{40}$ In addition, $20-\mathrm{OH}-\mathrm{LTB}_{4}$ and 12hydroxyeicosatetraenoic acid [12(R)-HETE] bind to BLT1. Moreover, the pro-resolving lipid resolvin E1 uses BLT1 to promote neutrophil apoptosis and the resolution of inflammation. ${ }^{41}$ The receptor is expressed predominantly by leukocytes of the innate immune system, such as neutrophils, mast cells, monocytes, macrophages, basophils, eosinophils and dendritic cells. ${ }^{16}$ The expression level of BLT1 on neutrophils is modulated by complex and poorly defined environmental cues. Prestimulation with CXCR1/2 ligands attenuates $\mathrm{LTB}_{4}$-induced $\mathrm{Ca}^{2+}$ signaling and the chemotaxis of human neutrophils in vitro. ${ }^{42}$ In vivo, however, BLT1 and CXCR2 cooperate to drive neutrophils into the skin of mice suffering from imiquimod-induced psoriasis. ${ }^{43}$ Disruption of BLT1 signaling enhances the internalization of FPR1 and C5aR1, thereby decreasing neutrophil chemotaxis towards $\mathrm{FMLF}$ and $\mathrm{C} 5 \mathrm{a}^{44}$

It is important to note that the contributions of BLT1 signaling during neutrophil recruitment in vivo have been unraveled to a lesser extent than those of other classical neutrophil chemoattractant receptors, i.e., CXCR1/2, FPR1/2 and C5aR1. Accumulating evidence suggests that fluctuations in BLT1 expression levels on neutrophils may be interesting from a clinical point of view and for targeting $\mathrm{LTB}_{4}-\mathrm{BLT1}$ interactions (Supplementary Table 1). In mice, BLT1-driven pathological neutrophil recruitment has been implicated in multiple inflammatory diseases, including skin inflammation, arthritis and acute kidney injury. ${ }^{45-48}$ In contrast, a protective function of $\mathrm{LTB}_{4}-\mathrm{BLT1}$ signaling was evidenced in a murine model of acetaminophen-induced liver toxicity. ${ }^{49}$ Specifically, the accumulation and activation of hepatic neutrophils were increased in BLT1-deficient mice, which developed more pronounced disease symptoms. The fact that the $\mathrm{LTB}_{4}-\mathrm{BLT} 1$ axis is important for neutrophil migration, as well as for activation, illustrates the important role of this ligand-receptor pair in facilitating pathogen clearance during polymicrobial sepsis. ${ }^{50}$ However, the resulting excessive inflammation eventually leads to enhanced mortality.

Notably, a second receptor that binds $\mathrm{LTB}_{4}$ has been identified, although it has low affinity, i.e., BLT2. ${ }^{51}$ Compared to BLT1, BLT2 is more ubiquitously expressed, shows a certain degree of promiscuity and transduces signals in response to multiple eicosanoids. The current knowledge on the precise biochemical and functional characteristics of BLT2 is limited. However, LTB L $_{4}$ BLT2 signaling may be involved in neutrophil migration.

PAFR. PAFR is expressed in most organs, muscles and leukocytes, including neutrophils. ${ }^{52}$ The receptor is activated by PAF, a highly potent proinflammatory phospholipid with neutrophilchemotactic and neutrophil-activating properties at picomolar concentrations. Several biomolecules act as natural PAFR agonists and show PAF-like activity, including LPS, oxidized low-density lipoprotein, and streptococcus-derived lipoteichoic acid moieties. ${ }^{52}$ Harmful effects of PAFR-driven neutrophil recruitment and inflammation were observed in mouse models of folic acid-induced renal inflammation, sponge-induced granuloma, intestinal ischemia-reperfusion-related inflammation, influenza
A-related lung injury and zymosan-induced joint inflammation (Supplementary Table 1). ${ }^{53-57}$ In addition, PAFR-dependent neutrophil trafficking has been implicated in cancer. For instance, inhibition of PAFR following coinjection of a subtumorigenic dose of melanoma cells and apoptotic cells in mice attenuated neutrophil and macrophage infiltration, eventually leading to impaired tumor growth. ${ }^{58}$ Due to its potent activation of neutrophil-dependent pathogen killing, PAFR may also fulfill defensive functions. A protective role for PAFR-driven neutrophil migration and activation was found during K. pneumonia-induced lung infection, with PAFR-deficient mice showing increased neutrophil-mediated bacterial clearance and impaired survival. ${ }^{59}$ Similarly, PAFR signaling is essential for the host defense against $P$. aeruginosa pneumonia, as demonstrated by the fact that lung inflammation and injury are increased and the phagocytic capacity of neutrophils is impaired in the absence of PAFR. ${ }^{60}$

\section{Complement receptors}

C3aR. The complement receptor C3aR is mostly expressed by leukocytes of myeloid lineages, including neutrophils, monocytes, eosinophils, basophils and mast cells. ${ }^{19}$ However, it has been suggested that basophils and eosinophils are the principal target cells for $\mathrm{C} 3 \mathrm{a}$ within peripheral blood. The precise function of $\mathrm{C} 3 \mathrm{aR}$ and its response to $\mathrm{C} 3 \mathrm{a}$ depend on the cell type studied. In mice, circulating LPS promotes the upregulation of C3aR on neutrophils and activation of the complement cascade, thereby facilitating NETosis and pushing neutrophils towards a protumoral phenotype. ${ }^{61}$ Interestingly, costimulation with its downstream cousin C5a inhibits C3a-induced internalization of C3aR expressed by human neutrophils. ${ }^{62}$ Regarding the functional role of the C3aR expressed by neutrophils, contradictory results have been published, and opposite functions have been attributed to C3a. Compelling scientific evidence suggests that C3a does not directly induce neutrophil chemotaxis, despite its potency to induce the activation of ERK $1 / 2$ and Akt-dependent pathways in these cells. ${ }^{63}$

When interpreting the results of $\mathrm{C} 3 \mathrm{a}$ activity on neutrophils, one should consider that certain described effects are most likely secondary to its effects on eosinophils that may be present in neutrophil preparations. Indeed, supernatant from C3a-stimulated eosinophils promotes neutrophil chemotaxis. ${ }^{64}$ Genetic ablation and pharmacological inhibition of $\mathrm{C} 3 \mathrm{aR}$ in vivo have been associated with increased and impaired neutrophil migration and may have beneficial or harmful consequences, depending on the experimental model (Supplementary Table 1). In the case of the increased number of neutrophils upon deletion of $\mathrm{C} 3 \mathrm{aR}$, it remains to be determined whether this reported augmentation of neutrophil recruitment results from compensation by other chemoattractant receptors and their cognate ligands. Interestingly, C3aR-deficient mice showed an enhanced susceptibility to granulocyte colony-stimulating factor (G-CSF)-induced neutrophil mobilization, and $\mathrm{C} 3 \mathrm{aR}$ stimulation suppressed neutrophil mobilization in wild-type mice. Follow-up research revealed that, upon the introduction of spinal cord injury in the mice, C3aR signaling antagonized CXCR2-induced neutrophil bone marrow egress via PTEN. ${ }^{65}$ Moreover, fewer circulating neutrophils in humans with spinal cord injury correlate with improved recovery, suggesting that $\mathrm{C} 3 \mathrm{aR}$ and PTEN might be interesting therapeutic targets in this context.

C5aR1. The complement activation product $\mathrm{C} 5 \mathrm{a}$ is an exceptionally powerful mediator of inflammation and has potent neutrophil chemotactic activity. The receptor recognized by this anaphylatoxin, $\mathrm{C} 5 \mathrm{aR} 1$, is expressed by virtually every cell type, including leukocytes (e.g., neutrophils, mast cells, monocytes and eosinophils) and nonleukocytes, such as epithelial and endothelial cells, synoviocytes, keratinocytes, astrocytes and cardiomyocytes. ${ }^{19}$ Various cell types, including neutrophils, express a second receptor for $\mathrm{C} 5 \mathrm{a}$, i.e., C5L2 or C5aR2, which shows no detectable 
G protein coupling upon ligand stimulation but functions presumably as an important regulator of C5aR1-mediated signaling. $^{66}$ It was recently demonstrated that C5aR1-mediated neutrophil arrest in murine arthritis in vivo requires the initial transport of $\mathrm{C} 5 \mathrm{a}$ into the vessel lumen, which is facilitated by endothelial cell-expressed C5L2. ${ }^{67}$ The stimulation of human neutrophils with $\mathrm{C} 5 \mathrm{a}$ results in rapid internalization of $\mathrm{C} 5 \mathrm{aR} 1$, followed by re-expression of the receptor. Moreover, preincubation with $\mathrm{fMLF}$ desensitizes C5a-induced chemotaxis of human neutrophils and vice versa, without affecting the ROS release or degranulation. ${ }^{68}$ The cytokines TNF- $a$ and granulocytemacrophage colony-stimulating factor (GM-CSF) also attenuate C5a-induced neutrophil chemotaxis. ${ }^{69}$

Altered $\mathrm{C} 5 \mathrm{aR} 1$ expression in patient neutrophils may be of clinical significance. For example, neutrophils from septic patients showed reduced C5aR1 expression and impaired C5a-induced chemotaxis during the early phase of the disease. ${ }^{70}$ During late stages of the disease, re-expression of $\mathrm{C} 5 \mathrm{aR} 1$ on neutrophils was found and reflected the recovery of neutrophil activity. Although low expression of $\mathrm{C} 5 \mathrm{aR} 1$ on neutrophils is indicative of mortality, inhibition of either $\mathrm{C} 5 \mathrm{a}$ or $\mathrm{C} 5 \mathrm{aR} 1$ improves survival in experimental sepsis. ${ }^{71}$ Similarly, neutrophils from trauma patients displayed reduced $\mathrm{C} 5 \mathrm{aR} 1$ expression, with $\mathrm{C} 5 \mathrm{aR} 1$ expression levels being inversely correlated with injury severity. ${ }^{72}$ In HIV patients, decreased C5aR1 expression on neutrophils was reported and accompanied by impaired $\mathrm{C} 5 \mathrm{a}$-induced migration and degranulation. ${ }^{73}$ Neutrophils found in bronchoalveolar lavage fluids from healthy volunteers or patients with sarcoidosis also displays an activated phenotype, as indicated by reduced $\mathrm{C} 5 \mathrm{aR} 1$ expression. $^{74}$ In this case, an additional decrease in C5aR1 expression was detected in the neutrophils from the patients. Likewise, neutrophils from patients with chronic renal failure showed reduced $\mathrm{C} 5 \mathrm{a}$-binding capacity. ${ }^{75}$ Although most studies on the expression of $\mathrm{C} 5 \mathrm{aR} 1$ on patient neutrophils reported decreased expression, human neutrophils transmigrated into skin blisters, expressed increased levels of $\mathrm{C} 5 \mathrm{aR} 1$ and an enhanced chemotactic response to $\mathrm{C} 5 \mathrm{a} .{ }^{76}$

Clearly, targeting C5aR1 signaling may be a relevant approach to limit excessive neutrophil recruitment and activation. The results from intense research efforts favor this hypothesis (Supplementary Table 1). In mice, C5aR1-deficient neutrophils failed to induce arthritis, but initiation of local inflammation by wild-type neutrophils paved the way for the entrance of these cells. ${ }^{77}$ Genetic ablation of murine C5aR1 corresponded to a strongly reduced susceptibility to the development of antibodyinduced arthritis, and anti-C5aR1 treatment was associated with decreased neutrophil infiltration in the early phase of murine delayed-type hypersensitivity arthritis. ${ }^{78,79}$ C5aR1 signaling presumably also plays an important role during neutrophil migration in human arthritis. ${ }^{80}$

C5aR1 inhibition seems highly relevant in the context of ischemia-reperfusion injury. For example, inhibition of C5aR1 impaired neutrophilia and neutrophil infiltration in hepatic ischemia-reperfusion injured tissue and was associated with an attenuated release of inflammatory mediators and survival. ${ }^{81}$ In a model of anti-neutrophil cytoplasmic autoantibody-induced necrotizing crescentic glomerulonephritis, the numbers of glomeruli-infiltrating neutrophils and level of albuminuria were significantly reduced in the mice that had received bone marrow transplants from C5aR1-deficient littermates. ${ }^{82}$ The absence or inhibition of $\mathrm{C} 5 \mathrm{aR} 1$ also attenuated anti-neutrophil cytoplasmic antibody-induced neutrophil dysfunction and injury in murine glomerulonephritis, reduced the infiltration of neutrophils into the kidneys and prevented progressive impairment of renal functions in mice with lupus nephritis. ${ }^{83,84}$ Treatment with anti-C5aR1 reduced the number of neutrophils in bronchoalveolar lavage fluid and was associated with amelioration of airway hyperresponsiveness in mice. ${ }^{85}$ Similarly, C5aR1-targeted therapy reduced ovalbumin-induced neutrophil influxes and airway inflammation in a murine model of allergic asthma. ${ }^{86}$ Finally, a pivotal role for C5a-C5aR1 signaling during pathological neutrophil recruitment in the context of high-fat diet-induced vascular inflammation in mice was revealed. ${ }^{87}$

Formyl peptide receptors

FPR1 and FPR2. FPRs are expressed by immune and nonimmune cells, though they are predominantly recognized as myeloid chemoattractant receptors. ${ }^{20-22}$ Human neutrophils express FPR1 and FPR2, which are well-known for their unusual degree of promiscuity and pleiotropic action (Table 1). These classical GPCRs display homologous desensitization upon agonist stimulation, which is nullified upon activation of the ATP receptor P2Y2, but not vice versa. ${ }^{88}$ FPR1 was the first human neutrophil chemoattractant receptor to be cloned and is the high-affinity receptor for the prototypical formyl peptide $\mathrm{fMLF}$, which is abundant in the supernatant of $E$. coli cultures. Formyl peptides of different bacterial origins were identified and are now considered collectively as PAMPs. Regarding the chemotaxis of human neutrophils, fMLF-induced migration predominates over CXCL8or $\mathrm{LTB}_{4}$-induced chemotactic cues in vitro. ${ }^{89}$ This superior potency of $\mathrm{fMLF}$ is enhanced in the presence of LPS, which increases FPR1 expression while inducing internalization of CXCR1/2, BLT1 and C5aR1. ${ }^{90}$ In contrast to LPS, IL-4 inhibits the expression of FPR 1 on neutrophils. ${ }^{91}$ Alterations in FPR1/2 expression levels on neutrophils have been implicated in disease (Supplementary Table 1). For instance, significant upregulation of FPR1 was observed on tumor-infiltrating neutrophils in primary human colorectal cancer samples. $^{92}$

It is worth mentioning that several medical drugs interfere with FPR functions. Cyclosporins, for example, exhibit FPR1 inhibitory activity. ${ }^{93}$ Similarly, piroxicam and propofol bind to FPR1 to inhibit fMLF-induced neutrophil responses. ${ }^{94,95}$ Additionally, distinct pathogen-related molecules may influence FPR signaling or expression. These proteins include chlamydial protease-like activating factor (CPAF), which paralyses neutrophils by cleaving surface FPR2, and S. aureus-derived FPRL1 inhibitory protein (FLIPr), which disrupts FPR2-mediated $\mathrm{Ca}^{2+}$ signaling and neutrophil chemotaxis. ${ }^{21,96}$

FPRs are well known for their capacity to recognize and respond to mitochondrial-derived DAMPs. Thus, persistent neutrophil activation enables the maintenance of inflammation via the release of DAMPs. Mitochondrial DAMPs induce $\mathrm{Ca}^{2+}$ signaling and MAPK activation in human neutrophils, mediate chemotaxis and activation of the oxidative burst, promote the release of MMP9 and CXCL8 by these cells, and may suppress cellular responses to CXCL1, $\mathrm{LTB}_{4}$ and $\mathrm{fMLF}^{97,98}$ Mitochondrial DAMPs were abundantly present in bronchoalveolar fluids and serum from patients with acute respiratory distress syndrome, and deficiency or inhibition of FPR1 resulted in reduced inflammation in a murine model of this disease. ${ }^{99}$ Additionally, high levels of mitochondrial DAMPs were associated with the development of primary graft dysfunction in lung transplantation patients. ${ }^{100}$ Moreover, analysis of primary graft dysfunction in a mouse lung transplantation model revealed a pivotal role for FPR1-driven neutrophil trafficking and activation in this context.

Direct interactions have been demonstrated between FPR2 and resolvin D1, a metabolic byproduct of the omega-3 fatty acid docosahexaenoic acid. ${ }^{21}$ As indicated by its name, resolvin D1 has pro-resolving, anti-inflammatory functions. This lipid cooperates with FPR2 to block neutrophil recruitment and neutrophilic inflammation in vivo. For instance, the resolvin D1-FPR2 axis has been implicated in LPS-induced lung injury, with resolvin D1-FPR2 signaling reducing CXCL2 expression in alveolar macrophages to attenuate neutrophil accumulation and protect mice from acute lung injury. ${ }^{101}$ FPR2 was originally considered a high-affinity receptor for the pro-resolving arachidonic acid derivative lipoxin 
440

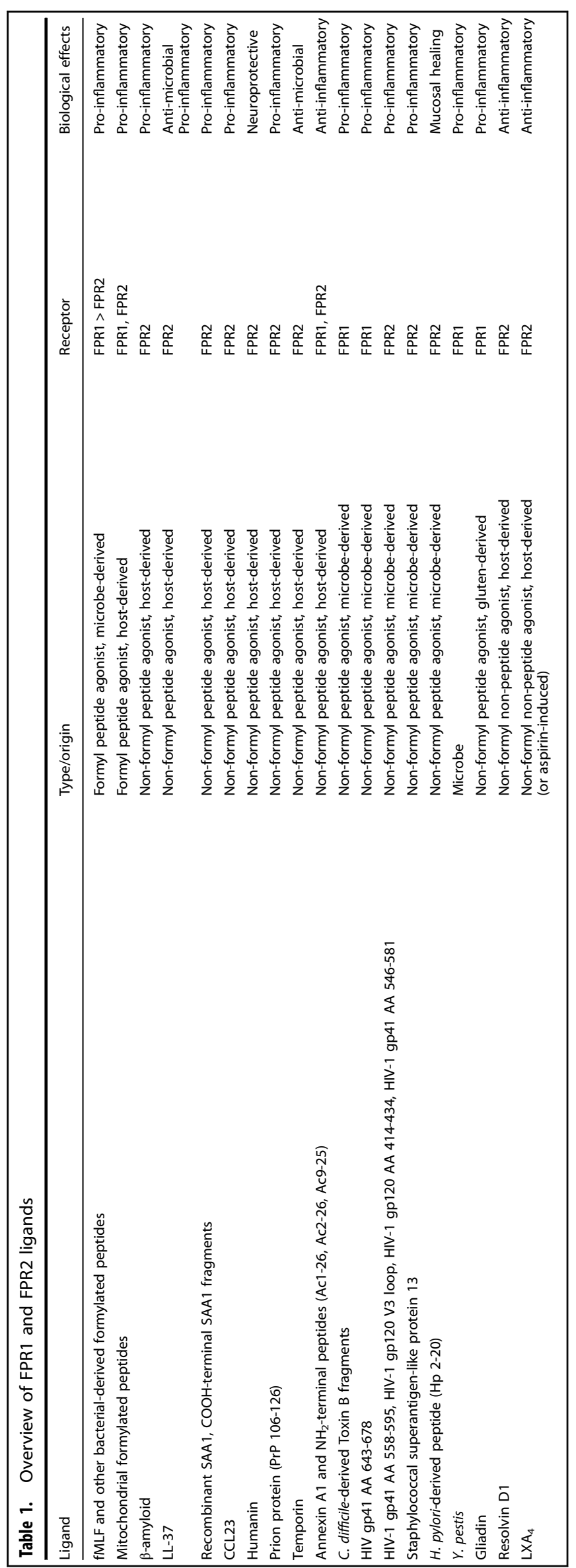

A4 ( $\left.\mathrm{LXA}_{4}\right)$, which has anti-adhesive effects and inhibits neutrophil transmigration. ${ }^{40}$ However, several research groups observed that $\mathrm{LXA}_{4}$ and FPR2 had no interaction, and the incorrect use of FPR inhibitors has been reported. ${ }^{21}$ Consequently, persuasive scientific evidence concerning the identity of the receptor used by LXA $\mathrm{L}_{4}$ to exert its pro-resolving functions in vivo is lacking. Apparently conflicting results were also published in the context of SAA-FPR2 interactions. Indeed, it was initially suggested that SAA induces $\mathrm{Ca}^{2+}$ signaling and chemotactic responses in neutrophils via FPR2. However, although commercially available SAA shows FPR2 agonistic activity, this recombinant protein is most likely no valid substitute for the natural, endogenously expressed molecule. ${ }^{102}$ It is also important to point out that many studies on the SAA-FPR2 axis depended on a SAA1-SAA2 hybrid that does not exist in humans. Nevertheless, we recently demonstrated that SAA fragments used FPR2 to synergize with CXCL8 in neutrophil chemotaxis assays. $^{103}$

An interesting ligand that uses all human FPRs is annexin A1, a $\mathrm{Ca}^{2+}$-dependent phospholipid-binding protein with potential antiinflammatory activity. Annexin $\mathrm{A} 1$ and its $\mathrm{NH}_{2}$-terminal peptides Ac1-26 and Ac9-25 elicit $\mathrm{Ca}^{2+}$ responses in human neutrophils via FPR1 without fully activating MAPK-dependent signaling. ${ }^{104}$ Genetic ablation of annexin A1 in murine neutrophils accelerated their maturation and reduced the chemotactic responses to CXCL12. ${ }^{105}$ Moreover, annexin A1 deficiency or FPR2 inhibition resulted in increased numbers of circulating $\mathrm{CXCR} 4^{+}$neutrophils, suggesting a role for annexin $A 1$ in neutrophil homing and maturation via the modulation of CXCL12-CXCR4 signaling.

Due to their pleiotropic nature and ligand variety, proinflammatory and anti-inflammatory functions have been attributed to FPRs (Supplementary Table 1). In a mouse model of $L$. monocytogenes infection, the absence of functional FPR1/2 resulted in impaired neutrophil mobilization during early infection, thereby worsening disease and increasing mortality. ${ }^{106}$ FPR1/2 exerts protective effects during bacterial meningitis, as demonstrated by the observation that FPR1/2-deficient mice suffer from increased bacterial burden, enhanced neutrophil infiltration and lower survival. ${ }^{107}$ The absence of FPR2 resulted in the excessive release of NETs and higher mortality in mice with severe infectioninduced lung injury. ${ }^{108}$ Moreover, upon the introduction of a sterile skin injury in mice, FPR1/2 promoted rapid neutrophil infiltration to facilitate wound healing. ${ }^{109}$ In contrast, intravital imaging revealed that FPR1 drove pathological neutrophil infiltration into the liver in a murine model of hepatic ischemiareperfusion injury. ${ }^{110}$ Beneficial effects of FPR1 deficiency or neutralization and the subsequent inhibition of neutrophil recruitment were observed in murine models of cigarette smoke-induced emphysema, endotoxin-induced lung inflammation, endometriosis and experimental colitis. ${ }^{11-114}$ In addition, carbon monoxide-mediated interference of FPR1 signaling led to reduced neutrophil infiltration in and promoted the survival of septic mice. ${ }^{115}$

\section{Chemokine receptors}

CXCR1. CXCR1 is a key receptor for the trafficking of neutrophils. ${ }^{24,116}$ The human receptor is activated upon stimulation with CXCL6 and CXCL8, whereas CXCL5 exhibits weak CXCR1 agonistic activity. ${ }^{117}$ Moreover, the $S$. aureus-derived leukotoxin ED utilizes CXCR1 to induce the death of neutrophils in vitro. ${ }^{118}$ Although neutrophils are the predominant CXCR1-expressing cells, a range of other cell types, including mast cells, macrophages, osteoclasts, CD8-positive effector T cells, natural killer (NK) cells, basophils and cancer cells, may express detectable levels of functional CXCR1. ${ }^{24}$ The expression level of CXCR1 on neutrophils is life cycle-dependent, with naive neutrophils being characterized by high CXCR1 expression and activated/infiltrated cells displaying low CXCR1 expression (Fig. 4). Phagocytosing neutrophils, for example, are characterized by reduced CXCR1 expression. ${ }^{119}$ 


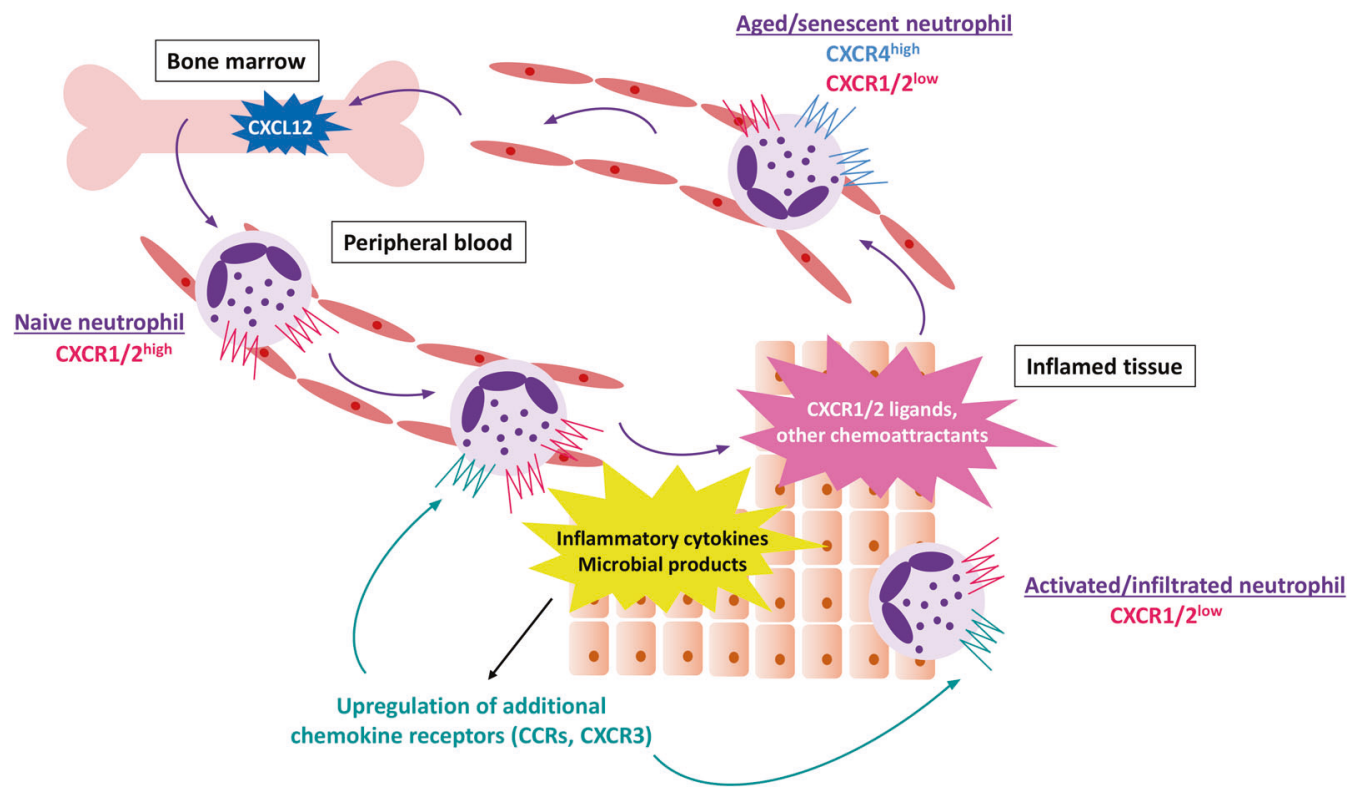

Fig. 4 Regulation of chemokine receptor expression by neutrophils. Naive neutrophils, freshly released from the bone marrow, display high levels of CXCR1 and CXCR2, which are the two major chemokine receptors involved in the trafficking of human neutrophils. Exposure to endogenous or exogenous inflammatory mediators may induce the upregulation of additional chemokine receptors, e.g., CCRs and CXCR3. Prolonged stimulation or exposure to high chemokine concentrations induces the downregulation of chemokine receptors. Consequently, highly activated, infiltrated cells are characterized by low CXCR1 and CXCR2 expression levels. Neutrophil aging is accompanied by the upregulation of CXCR4 expression, thereby facilitating a return of the cell to the bone marrow, which contains high levels of CXCL12

Upon execution of their effector functions at the site of inflammation, neutrophils may re-enter the vasculature/migrate away from the inflamed tissue, a phenomenon known as reverse transendothelial migration. ${ }^{120}$ Low CXCR1 expression is a hallmark of these reverse-transmigrated neutrophils. CXCR1 on neutrophils may also be downregulated in response to inflammatory agents such as TNF-a, Toll-like receptor 2 (TLR2) and TLR4 agonists, $H$. pylori and elastase or upon reoxygenation. ${ }^{121-125}$ In addition, exposure to body fluids from patients with inflammatory pathologies induces a rapid downregulation of CXCR1 on healthy donor neutrophils. For example, reduced CXCR1 expression was observed in healthy donor neutrophils incubated with the plasma from patients with alcoholic hepatitis. ${ }^{126}$ Interestingly, inhibitors of TLR2, TLR4, and TLR9 signaling prevented the downregulation of CXCR1 expression on neutrophils. Similarly, stimulation of healthy donor neutrophils with IL-4/IL-13 or exposure to serum from allergic patients reduced CXCR1 expression, accompanied by reduced CXCL8-induced migration and an impaired ability to release NETs. ${ }^{127}$ The fact that administration of LPS in healthy volunteers led to decreased CXCR1 levels on human neutrophils and a threefold increase in the release of ROS production illustrates the potential functional relevance of the modulation of CXCR1 expression in vivo. ${ }^{128}$

In line with the reported downregulation of CXCR1 on activated/infiltrated neutrophils, multiple research groups reported reduced CXCR1 expression on neutrophils from patients with various diseases (Supplementary Table 1). For example, decreased CXCR1 expression was observed on infiltrated neutrophils from asthmatic and chronic obstructive pulmonary disease (COPD) patients compared to the expression on blood neutrophils. ${ }^{129}$ Moreover, proteolytic activity in the lungs of cystic fibrosis patients and, to a lesser extent, in lungs of COPD patients, induced the cleavage of CXCR1 on neutrophils, thereby impairing CXCL8-induced bacterial killing. ${ }^{130}$ Low CXCR1 expression on neutrophils was associated with end-stage renal disease or acute pyelonephritis in children. ${ }^{131,132}$ Furthermore, a correlation was found between reduced CXCR1 levels on patient neutrophils and the disease severity of hepatitis B-related liver failure, suggesting a potential role for CXCR1 as a predictive biomarker in this context. ${ }^{133}$ Interestingly, neutrophils from patients with chronic kidney disease displayed reduced CXCR1 expression, with the neutrophils from nonresponders to recombinant human erythropoietin therapy showing a more pronounced decrease in CXCR1 levels. ${ }^{134}$ In contrast, CXCR1 was greatly upregulated in the kidneys of candidiasis-infected mice, and CXCR1 deficiency resulted in decreased survival and promoted kidney failure. ${ }^{135}$ Moreover, mice lacking CXCR1 displayed enhanced susceptibility to the development of systemic candidiasis due to defective pathogen killing by neutrophils. Thus, the precise CXCR1 levels most likely depend on the local (inflammatory) environment and the location in the body (tissue-infiltrated cells versus bloodstream-circulating cells), with important roles in the neutrophil life cycle.

CXCR2. CXCR2 is a major chemokine receptor expressed by neutrophils. ${ }^{24,116}$ Perceptible CXCR2 expression was also detected in other cell types, including keratinocytes, monocytes and a variety of cancer cells. The human receptor is activated by all seven ELR-positive CXC chemokines, i.e., CXCL1 to CXCL3 and CXCL5 to CXCL8, and is the putative receptor through which these chemokines exert their angiogenic activity. Interestingly, stimulation of CXCR2 in human neutrophils primes CXCL8-induced $\mathrm{Ca}^{2+}$ signaling via CXCR1, and it has been suggested that CXCR2 is an important receptor for responses to agonist at low concentrations. ${ }^{136}$ In addition to mediating neutrophil migration to the inflamed site, CXCR2 signaling promotes the release of neutrophils from the bone marrow, thereby antagonizing CXCR4-mediated bone marrow retention of neutrophils. ${ }^{137}$

CXCR2 is targeted by distinct bacterial products. For example, $S$. aureus-derived leukotoxin ED uses CXCR2 for killing neutrophils, and staphopain $A$ acts as a natural CXCR2 blocker. ${ }^{118,138}$ The expression of CXCR2 on neutrophils is modulated by environmental cues and related to their state of activation (Fig. 4). ${ }^{19}$ For example, stimulation with TNF- $a$, nitric oxide, TLR2 or TLR4 agonists, confrontation with $H$. pylori, low-dose prednisolone therapy or exposure to high chemokine ligand concentrations 
442

induced the downregulation of CXCR2 expression, whereas CXCR2 upregulation was promoted by neutrophil-derived lipocalin$2^{34,90,121,123,124,139-142}$ CXCR2 expression levels were enhanced upon stimulation with G-CSF, which simultaneously induced the downregulation of CXCR4, thereby promoting neutrophil mobilization into the blood circulation. ${ }^{127}$ To add an additional layer of complexity, the effect of an individual inflammatory mediator is context-dependent. For example, IL-4 counteracts the G-CSFinduced upregulation of CXCR2. ${ }^{127}$ Moreover, in mice, G-CSF facilitated neutrophil mobilization only at a relatively late stage of $E$. coli-induced acute inflammation, while it prevented excessive neutrophil egress during the early phase of inflammation by negatively modulating CXCR2-mediated signaling. ${ }^{143}$

In line with the reported downregulation of CXCR2 levels upon neutrophil activation, reduced CXCR2 levels were detected on neutrophils from patients with a range of immune-mediated diseases, with possible functional consequences (Supplementary Table 1). For example, low CXCR2 expression on peripheral blood neutrophils from patients with hepatitis $B$ virus-related acute-onchronic liver failure was related to a poor prognosis. ${ }^{133}$ In patients with systemic lupus erythematosus, an abnormal modulation of CXCR2 expression by neutrophils was observed and coincided with diminished CXCL8-induced response levels. ${ }^{144}$ Similarly, neutrophils from septic patients showed decreased CXCR2 levels and a suppressed CXCL8-induced migratory response. ${ }^{145,146}$ In a mouse model of sepsis, phospholipase D2 was critical for the downregulation of CXCR2 on neutrophils and inhibition of NET formation. $^{147}$ In contrast, upregulation of CXCR2 expression on neutrophils was detected during the relapse phase of ocular Behçet disease. ${ }^{142}$ It is important to note that changes in CXCR2 expression levels on neutrophils do not necessarily recapitulate the alterations in ligand-induced functional responses and vice versa. For example, peripheral blood neutrophils from patients with primary ciliary dyskinesia displayed unaltered CXCR2 expression but attenuated CXCR2-mediated chemotactic responses. ${ }^{148}$

Depending on the disease studied and the experimental model, CXCR2 is a potentially interesting drug target (Supplementary Table 1). In hypoxia-induced lung inflammation in mice, for example, deficiency of CXCR2 was linked to reduced neutrophil recruitment and ameliorated lung injury, which conferred a survival advantage. ${ }^{149}$ A beneficial effect of CXCR2 blockage or deficiency was reported in murine and rat models of sterile gut inflammation. ${ }^{150-152}$ Notably, the administration of anti-CXCR2 therapy in rats reduced the neutrophil infiltration only in the early phase of sterile colitis. ${ }^{151}$ Similarly, CXCR2 inhibition or deficiency had favorable effects in murine models of dsRNA-induced lung inflammation, in lung transplant ischemia-reperfusion injury, helminth-mediated keratitis, and in murine bronchiolitis obliterans syndrome. ${ }^{153-156}$ In humans, the administration of a specific CXCR2 antagonist elicited a reduction in circulating and infiltrated neutrophils in patients with severe asthma and inhibited LPSinduced neutrophilic inflammation in healthy volunteers. ${ }^{157,158}$ However, treatment with a selective CXCR2 blocker did not reduce the incidence of severely exacerbated asthma in patients. ${ }^{159}$

Due to the crucial role of neutrophils in pathogen defenses, inhibition of neutrophil recruitment and/or activation may have detrimental effects. For example, in murine models of $P$. aeruginosa-induced pneumonia, S. aureus-mediated experimental brain abscesses and E. coli-related kidney infection, CXCR2 deficiency led to reduced neutrophil recruitment and diminished pathogen killing. ${ }^{160-162}$ Moreover, CXCR2 blockade and the subsequent reduced neutrophil recruitment were associated with enhanced mortality in a mouse model of $L$. pneumophila-induced pneumonia. ${ }^{163}$ In contrast to the advantageous effects of CXCR2 blockage in sterile gut inflammation (vide supra), in a mouse model of $C$. rodentium-induced gut infection, an indispensable role for CXCR2 was demonstrated in the regulation of neutrophil recruitment, which facilitated pathogen clearance. ${ }^{164}$ Similarly,
CXCR2 deficiency led to enhanced susceptibility to herpetic stromal keratitis due to impaired neutrophil recruitment and, consequently, to a prolonged duration of virus infection in the eye. ${ }^{165}$ Furthermore, the absence of CXCR2 was associated with delayed experimental cutaneous wound healing in mice, supporting the notion that neutrophils are critically involved in tissue repair. ${ }^{166}$

Neutrophils within the tumor microenvironment may be classified into protumoral and antitumoral phenotypes, ${ }^{167}$ and the contributions of tumor-supporting neutrophils to the disease state are usually overwhelming. Certain molecules within the tumor microenvironment may promote the recruitment of protumoral neutrophils. For example, tumor-derived oxysterols interact with CXCR2 to attract protumoral neutrophils, with disruption of CXCR2-oxysterol interactions resulting in the attenuation of tumor growth and offering a survival advantage. ${ }^{168}$ CXCR2 and its chemokine ligands have been widely implicated in tumorigenesis and metastasis, and the inhibition of CXCR2 was found to be beneficial in distinct cancer models. In murine lung cancer, for example, CXCR2 blockage and the subsequent inhibition of neutrophil recruitment were associated with decreased tumor growth and even reduction of tumor size in vitro and in vivo. ${ }^{169,170}$ Deficiency of CXCR2 and the resulting attenuation of neutrophil infiltration also suppressed the formation of skin and intestinal tumors and adenocarcinoma in mice. ${ }^{171}$ Moreover, the upregulation of CXCR2 was observed on tumorsupporting neutrophils in murine pancreatic ductal adenocarcinoma, and CXCR2 signaling was required for the development of metastasis in this model. ${ }^{172} \mathrm{~A}$ pivotal role for CXCR2 in promoting metastasis was also revealed in murine breast cancer. ${ }^{173}$ These experimental findings support the idea that CXCR2 expression level may serve as a prognostic biomarker. Consistently, ex vivo analysis of human hepatocellular carcinoma tissues revealed an association between CXCR2 levels, neutrophil infiltration and poor prognosis. $^{174}$

CXCR3. Human CXCR3 is activated by the three interferon (IFN)$\gamma$-inducible chemokines CXCL9, CXCL10 and CXCL11 and is the presumed receptor for the platelet-derived chemokines CXCL4 and CXCL4L1. ${ }^{116,175}$ The receptor is abundantly expressed on activated T cells, regulatory T cells, CD4- and CD8-positive effector and memory $T$ cells but also on innate lymphoid cells, $\gamma \delta T$ cells, NK and NKT cells, specific B cell subsets and dendritic cells. CXCR3 is not expressed on naive, non-infiltrated neutrophils; however, pulmonary and synovial neutrophils isolated from patients with chronic lung inflammation or rheumatoid arthritis, respectively, expressed CXCR3. ${ }^{176}$ This receptor expression seems functionally relevant since ligand stimulation induced chemotaxis and promoted phagocytosis, respiratory burst and a-defensin production. Follow-up experiments revealed that exposure to TLR agonists induced CXCR3 expression on healthy donor neutrophils in vitro. Investigation of neutrophil-mediated severe lung inflammation of viral and nonviral origin in mice revealed a critical role for CXCR3 and its ligand CXCL10 in neutrophil recruitment. ${ }^{177}$ Specifically, functional CXCR3 was detected on neutrophils that had infiltrated inflamed lungs, and its expression on these neutrophils was proposed to depend on TLR4-TIR-domaincontaining adapter-inducing IFN- $\beta$ (TRIF) signaling. Additionally, neutrophils infiltrating the lungs of influenza-infected mice expressed CXCR3, and an important role for CXCR3 in neutrophil recruitment in a mouse model of $S$. typhimurium-induced colitis was suggested. ${ }^{178,179}$

CXCR4. CXCR4 is the exclusive GPCR for the constitutively produced chemokine CXCL12 and a coreceptor for HIV-1. ${ }^{26,116}$ The receptor is expressed by virtually all hematopoietic cells, vascular endothelial cells, astrocytes, neurons, microglia and many cancer cells. ${ }^{116}$ The first scientific publications on the expression of 
CXCR4 on neutrophils reported controversial data. However, high levels of Cxcr4 mRNA were detected in human neutrophils, and the expression of the CXCR4 protein on these cells was confirmed using a specific antibody. ${ }^{180}$ Interestingly, the synergistic action of CXCL12 and the major human neutrophil-attracting chemokine CXCL8 in mediating the chemotaxis of human neutrophils was reported. ${ }^{181}$ The expression levels of CXCR4 on neutrophils most likely depend on the experimental/environmental context. For example, the cytokines TNF- $a$, IFN- $a$, IFN- $\gamma$, GM-CSF and G-CSF counteracted CXCL12-induced functional responses in neutrophils by inducing the internalization and consequent downregulation of CXCR4. ${ }^{182,183}$ In contrast, the injection of an adrenocorticotrophic hormone induced upregulation of CXCR4 on murine neutrophils in vivo. ${ }^{184}$

Multiple lines of evidence suggest a pivotal role for the CXCL12CXCR4 axis in the regulation of neutrophil homeostasis (Figs. 1 and 4). ${ }^{185}$ In support of this idea, patients with the primary immunodeficiency syndrome WHIM (abbreviation of 'Warts, Hypogammaglobinemia, Infections, and Myelokathexis'), which is caused by gain-of-function mutations in the $\mathrm{Cxcr} 4$ gene, typically display abnormal bone marrow retention of neutrophils. ${ }^{186}$ Indeed, CXCR4 is a master regulator of neutrophil storage in the bone marrow and facilitates homing of circulating neutrophils back to the bone marrow following senescence. ${ }^{187}$ CXCR4 was found to be expressed preferentially on aged neutrophils and was upregulated during the aging of human neutrophils in vitro, independent of the presence of inflammatory mediators. ${ }^{187,188}$ It is noteworthy that a role for the microbiome was recently revealed showing the regulation of a pathological neutrophil subset by promoting neutrophil aging in a TLR- and myeloid differentiation factor 88-dependent manner. ${ }^{189}$ Upon return to the bone marrow in vivo, neutrophils are phagocytosed by bone marrow stromal macrophages, which promotes the release of G-CSF by these cells. ${ }^{190}$ Under homeostatic conditions, G-CSF regulates the levels of circulating neutrophils by selectively downregulating CXCR4 expression on bone marrow-derived human and murine myeloid cells of various lineages, thereby promoting the release of these cells into the blood circulation. ${ }^{191}$

CXCL12 exerts dual effects on circulating neutrophils. Specifically, this chemokine not only direct neutrophil migration but also enhances the neutrophil expression of TNF-related apoptosisinducing ligand (TRAIL) and its receptor, thereby destining these cells to undergo apoptosis upon arrival at the bone marrow. ${ }^{192}$ Using a mouse model of sterile liver inflammation, it was recently demonstrated that liver-infiltrated neutrophils may reenter the circulation to follow a preprogrammed trafficking route that includes a temporary delay in the lungs, where CXCR4 is upregulated, and the subsequent return to the bone marrow, where they undergo apoptosis. ${ }^{193}$ Furthermore, an elevated number of aged CXCR4-expressing neutrophils was detected in the lung vasculature compared to that in the peripheral circulation, and the physical interaction of lung neutrophils and local B cells was discovered. ${ }^{194}$ The absence of B cells induced the accumulation of aged neutrophils in the lungs and resulted in fibrotic lung disease, supporting the hypothesis that the lungs may function as an intermediate stopover where senescent neutrophils are regulated by $B$ cells to prevent tissue damage.

The potency of G-CSF, CXCL2 and L. monocytogenes to induce the release of neutrophils from the bone marrow in mice is negatively affected by the disruption of CXCL12-CXCR4 signaling, which indicates that the homing receptor and its unique ligand are involved in neutrophil mobilization under homeostatic as well as pathological conditions. ${ }^{195}$ The upregulation of CXCR4 expression by neutrophils was reported in different mouse models of LPS-induced lung inflammation and influenza-induced pneumonia. ${ }^{178,196}$ In follow-up experiments, a link between the activation of L-selectin and upregulation of CXCR4 on neutrophils in vitro was described. ${ }^{196}$ Moreover, a role for the CXCL12-CXCR4 axis in the modulation of the transition from the inflammatory response to the initiation of tissue repair was suggested. ${ }^{197}$ Recent experimental findings showed that stimulation with a low dose of LPS induced the upregulation of CXCR4 on neutrophils and the release of NETs, which in turn promoted the uptake of house dust mites by dendritic cells. ${ }^{198}$ In addition, the induction of allergic asthma by influenza infection or ozone exposure required the release of NETs by CXCR4-expressing neutrophils. Thus, CXCL12CXCR4 interactions are presumed to be highly relevant also in allergies. Enhanced CXCR4 expression was observed on neutrophils from patients with various lung and joint pathologies (Supplementary Table 1). Remarkably, preincubation with synovial fluid from rheumatoid arthritis patients amplified the desensitizing effect of CXCL12 on the fMLF-evoked respiratory burst of healthy human neutrophils, suggesting a beneficial function of CXCL12 in the context of joint inflammation. ${ }^{199}$ Other pathologies in which CXCR4-expressing neutrophils have been implicated include murine lupus with active nephritis, deficiency of the glucose-6phosphatase G6PC3 in humans and mice, murine polymicrobial sepsis, and ischemic stroke in patients. ${ }^{200-203}$ Recently, a novel neutrophil subset with high proangiogenic potential characterized by the expression of the integrin a4 subunit CD49d, vascular endothelial growth factor receptor 1 (VEGFR1) and CXCR4 was identified in mice and humans. ${ }^{204}$ Inhibition of the migration of these proangiogenic neutrophils into hypoxic tissues in a mouse model of angiogenesis resulted in diminished vessel formation without affecting the neutrophil population involved in the defense against pathogens. These findings suggest an opportunity to selectively targeting neutrophil-mediated angiogenesis without disrupting the antimicrobial immune response.

CCR1. Human CCR1 is a promiscuous receptor that interacts with CCL3, CCL3L1, CCL4, CCL5, CCL7, CCL8, CCL13, CCL14, CCL15, CCL16 and CCL23 and is expressed by a wide variety of cells, including astrocytes, macrophages, dendritic cells, monocytes, lymphocytes, granulocytes and platelets. ${ }^{116}$ Although human neutrophils express low, yet detectable, levels of CCR1 under steady-state conditions, initial observations suggested that these cells do not respond to CC chemokines, including CCR1 ligands. However, CCL15 was reported to induce CCR1-mediated $\mathrm{Ca}^{2+}$ flux and neutrophil chemotaxis in humans. ${ }^{205}$ Moreover, significant neutrophil infiltration was observed two hours after the intradermal administration of CCL3 in humans. ${ }^{206}$ Notably, these authors demonstrated that neutrophils in whole blood expressed functional CCR1 but not after isolation. The surface expression of CCR1 on neutrophils is strongly enhanced upon confrontation with inflammatory mediators, including GM-CSF, TNF- $\alpha$ and IFN- $\gamma$, and an important role for CCR1 in the recruitment of murine and human neutrophils has been found under certain (inflammatory) conditions (Supplementary Table 1). Increased CCR1 levels were detected on the infiltrated neutrophils of patients with acute respiratory distress syndrome. ${ }^{207}$ Moreover, the analysis of lung metastases in colorectal cancer patients revealed significantly shorter relapse-free survival for patients with CCL15-positive metastasis and showed that most CCR1-expressing cells within the metastatic lesions were tumor-associated neutrophils. ${ }^{208}$ In mice, CCR1 (among other CC chemokine receptors) was found to mediate the recruitment of neutrophils to postischemic tissues by controlling intravascular adherence and transmigration. ${ }^{209}$ The presumedly important function of CCR1 in regulating the intravascular adherence and transmigration of neutrophils was confirmed by in vivo imaging of the mouse cremaster muscle. ${ }^{210}$ Using CCR1-knockout mice, an important role for CCR1 was found in the extravasation of neutrophils into the kidneys during murine hemolytic uremic syndrome. ${ }^{211}$ In this model, neutrophil renal infiltration was significantly reduced and delayed in the CCR1deficient mice and was accompanied by a delayed release of the inflammatory cytokines TNF- $a$ and IL- 6 . A study on wound healing 
revealed the overexpression of CCR 1 in murine neutrophils within the wounded area. ${ }^{212}$ Strikingly, no alterations in wound healing were observed in the CCR1-knockout mice. The authors suggested that the fact that CCR1 was dispensable for wound healing may be explained by a certain degree of redundancy between chemokine receptors.

CCR2. Human CCR2 is activated upon stimulation with CCL2, CCL7, CCL8, CCL13 or CCL16, while CCL26 acts as a natural CCR2 antagonist. ${ }^{116}$ The receptor is expressed mainly on monocytes, basophils, T cells, NK cells and immature dendritic cells and historically not considered a receptor for the recruitment of neutrophils. However, over the past two decades, it became clear that murine and human neutrophils express functional CCR2, particularly during severe (infectious) inflammation. For example, functional CCR2 was found on murine neutrophils in a model of chronic inflammation. ${ }^{213}$ Several lines of evidence suggest a critical function for CCR2 in ischemia-related neutrophilic inflammation. An important role for CCR2-mediated neutrophil recruitment was found; for example, in a study on ischemia-reperfusion injury by trans-illumination microscopy of the cremaster muscle in mice. ${ }^{209}$ In a murine model of liver ischemia-reperfusion injury, neutrophil mobilization was dependent on CCR2 expression, which, in turn, required the activation of the TLR4 - p38 MAPK axis. $^{214}$ In a model of cerebral ischemia-reperfusion injury, CCR2 deficiency was associated with a fourfold reduction in neutrophil infiltration and impaired production of inflammatory cytokines, accompanied by a reduction in infarct sizes and significantly decreased blood-brain barrier permeability and brain edema. ${ }^{215}$

CCR2-expressing neutrophils are potentially relevant in the context of sepsis. For example, neutrophils isolated from septic mice express $\mathrm{Ccr} 2 \mathrm{mRNA}$, are capable of CCL2 and CCL3 binding, and display CCL2/3-induced chemotaxis. ${ }^{216}$ Consistently, blocking CCR2 resulted in diminished neutrophil recruitment in a mouse model of polymicrobial septic peritonitis. ${ }^{217}$ Moreover, neutrophils from septic patients displayed high levels of CCR2, a key receptor mediating the infiltration of neutrophils into remote organs. ${ }^{218} \mathrm{~A}$ link between illness and increased chemotactic responses of patient neutrophils to CCR2 ligands was discovered. High expression levels of responsive CCR2 were discovered on neutrophils from the blood of patients with early rheumatoid arthritis and on blood and bone marrow neutrophils from mice with antigen-induced arthritis. ${ }^{219}$ Therefore, a critical role for CCR2 has been proposed for the detrimental infiltration of neutrophils into the joints of rheumatoid arthritis patients with active disease.

CCR3. Human CCR3 interacts with the chemokine ligands CCL3L1, CCL4, CCL5, CCL7, CCL11, CCL13, CCL15, CCL24, CCL26 and CCL28 and serves as a functional coreceptor for specific strains of HIV-1 during infection. ${ }^{116,220}$ Strikingly, the three CXCR3interacting chemokines CXCL9 to CXCL11 exhibit natural CCR3 antagonistic activity at high ligand concentrations. ${ }^{221}$ CCR3 is expressed most abundantly on eosinophils and basophils. Additionally, CCR3 expression has been detected on Th2 cells, keratinocytes, platelets and airway epithelial cells. Although CCR3 is not typically found on neutrophils, IFN- $\gamma$ treatment was found to induce an efficient upregulation of $\mathrm{Ccr} 3$ mRNA in human neutrophils in a dose-dependent manner. ${ }^{222}$ Moreover, IFN- $\gamma^{-}$ treated neutrophils displayed specific binding sites for labeled CCL7 and showed functional migration in response to CCL3, CCL5, CCL7, CCL11 and CCL15. These findings highlight the supposition that locally produced inflammatory cytokines may alter the expression patterns of chemokine receptors on leukocyte subsets, thereby modulating the target cell repertoire of certain chemokines (Fig. 4). The clinical significance of this hypothesis is underscored by the observation that neutrophils in lungs or joints from patients with inflammatory lung diseases and rheumatoid arthritis express high levels of functional CCR3. ${ }^{176,207}$ The observation that cutaneous injection of CCL11 and CCL24 induced neutrophil recruitment in healthy volunteers also supports a role for CCR3 in the recruitment of human neutrophils in vivo. ${ }^{223}$

CCR5. Human CCR5, the receptor for CCL3, CCL3L1, CCL4, CCL5, CCL8, CCL14 and CCL16 and for the antagonist CCL7, is predominantly expressed on monocytes and dendritic cells but also on endothelial cells, epithelial cells, astrocytes and vascular smooth muscle cells. ${ }^{116}$ In addition to interacting with its chemokine ligands, CCR5 acts as a major coreceptor for HIV-1 during infection and is a receptor for the $S$. aureus-derived leukotoxin ED. ${ }^{116,224}$ Constitutive expression of detectable CCR5 on freshly purified human neutrophils has been reported. ${ }^{225}$ Nevertheless, CCL3-induced phosphorylation of ERK1/2 via CCR5 required pretreatment of the neutrophils with GM-CSF. Additionally, GM-CSF stimulation facilitated neutrophil migration in response to CCL3 and CCL4 in a CCR5-dependent fashion. Regarding the relevance of CCR5-expressing neutrophils in vivo, a role for CCR5 was revealed in the recruitment of murine neutrophils after the induction of ischemia-reperfusion injury, upon acute endotoxin-induced lung injury and during infectious pneumonia. ${ }^{111,178,209}$ Furthermore, expression of CCR5 on apoptotic neutrophils (and activated apoptotic $T$ cells) during the resolution phase of murine peritonitis facilitated the scaffolding of CCL3 and CCL5, supporting the hypothesis that CCR5-expressing apoptotic leukocytes may be involved in the termination of chemokine signaling. ${ }^{226}$ An analysis of neutrophils from rheumatoid arthritis patients revealed the upregulation of C Cr5 gene expression, and CCR5 was detected on the infiltrated neutrophils from patients with chronic lung or joint inflammation and on tumor-associated neutrophils derived from lung cancer patients. ${ }^{176,227,228}$ The in vitro expression of CCR5 on human neutrophils was suggested to be apoptosis-related. ${ }^{176}$

ACKRs and CCRL2. In contrast to conventional chemokine receptors, ACKRs lack or show profound alterations in the canonical DRYLAIV motif in the second intracellular loop and are unable to couple to G proteins. In humans, four ACKRs (ACKR1 to ACKR4) that share the capacity to internalize chemokinespredominantly via $\beta$-arrestin-dependent mechanisms-as a hallmark feature were identified. ${ }^{116,229-231}$ Depending on the specific ACKR, the cellular context and likely also on the chemokine ligand considered, internalization may or may not be followed by activation of alternative signaling pathways. Among the four human ACKRs, ACKR1 and ACKR2 play important roles in modulating neutrophil trafficking and functional responses.

ACKR1, originally named 'Duffy antigen receptor for chemokines' (DARC), completely lacks the DRYLAIV motif. ${ }^{229-231}$ The receptor is expressed at high levels by erythrocytes and has been detected on stromal cells but is absent on leukocytes. In red blood cells, ACKR1 functions as a scavenger for CCR1, CCR2, CCR3, CCR4, CCR5, CXCR1, CXCR2 and CXCR3 ligands. ${ }^{230,231}$ Due to its potent ability to control the transport and biodistribution of these chemokines, the receptor is presumably involved not only in the modulation of inflammatory responses but also in the regulation of neutrophil homeostasis. The fact that the reduced number of neutrophils observed in individuals of African origin is strongly associated with null polymorphic ACKR1 favors this hypothesis. ${ }^{230}$ Intravital imaging of mice revealed that neutrophils facilitate their own apical-to-basolateral transmigration via the production of CXCL2, which is sequestered and presented by endothelial ACKR1. ${ }^{14}$ Importantly, these authors demonstrated that CXCL1 and CXCL2, produced by TNF-a-treated endothelial cells/pericytes and neutrophils, respectively, act in a sequential manner to promote neutrophil crawling (CXCL1) and breaching of endothelial junctions (CXCL2), supporting the notion that individual CXCR1/2 ligands may serve unique, nonredundant functions during in vivo neutrophil trafficking. Various results have been 
published regarding the effects of ACKR1 deficiency on neutrophil function and migration in vivo. The absence of the atypical receptor most likely affects in vivo neutrophil chemotaxis without affecting the susceptibility to bacterial infection. ${ }^{232}$ Notably, it remains to be determined whether the described phenotypes can be attributed to the loss of ACKR1 on erythrocytes or to the lack of ACKR1 expression by endothelial cells. In mice, the plasma concentrations of CXCL1 and CXCL2 were enhanced in the presence of CXCL5, which occupied ACKR1 on the erythrocytes to prevent scavenging by the two other CXCR1/2 ligands. ${ }^{233}$ The observed competition for ACKR1 binding may have important functional consequences, particularly during inflammation. Indeed, the absence of CXCL5 has been linked to increased neutrophil recruitment and decreased pathogen burden and a survival benefit during murine $E$. coli-induced pneumonia. ${ }^{233}$

ACKR2, also known as 'D6', contains a modified DRYLAIV motif ('DKYLEIV') and induces $\beta$-arrestin-dependent scavenging of inflammatory CCR1-, CCR2-, CCR3-, CCR4- and CCR5-interacting chemokines, which contributes to the resolution of inflammation. ${ }^{229,230}$ The receptor is expressed by trophoblasts, lymphatic endothelial cells, and distinct leukocyte subsets, such as alveolar macrophages and innate-like B cells. ${ }^{234}$ In addition, low ACKR2 levels were detected on murine neutrophils. A critical role for ACKR2 in fine-tuning neutrophil positioning during inflammation has been proposed. ACKR2-deficient mice develop a psoriasislike phenotype characterized by the uncontrolled misplacement of neutrophils into inflamed skin. ${ }^{235}$ Interestingly, genetic ablation of ACKR2 in neutrophils ameliorates their responsiveness to CCR1 stimulation. A protective role for ACKR2 was recently uncovered in the context of sepsis. Specifically, neutrophil accumulation in the lungs, kidneys and hearts of septic mice is increased in the absence of ACKR2, accompanied by elevated local concentrations of proinflammatory CC chemokines and enhanced severity of lesions. ${ }^{236}$ In the wild-type littermates, the expression of ACKR2 in these organs was increased in animals suffering from nonsevere sepsis compared to the expression in those with severe disease. Moreover, elevated numbers of ACKR2-expressing cells were detected in the lungs of septic patients. In the context of cancer development and progression, ACKR2 may fulfill harmful roles, with genetic ablation of the receptor promoting neutrophil mobilization, enhancing neutrophil-dependent tumor killing and conferring neutrophil-mediated protection against metastasis. ${ }^{237}$

CC chemokine receptor-like 2 (CCRL2) is expressed by connective tissue cells and various leukocyte subtypes, including neutrophils. $^{238}$ The receptor is recognized by chemerin-a chemotactic protein-and differs from the prototypical ACKRs in that it shows no chemokine scavenging activity and does not couple to $\beta$-arrestins. CCRL2 forms functional heterodimers with CXCR2, thereby regulating CXCR2 expression levels on neutrophils and modulating chemokine-induced, CXCR2-dependent signaling. ${ }^{239}$ Moreover, genetic ablation of CCRL2 results in defective neutrophil recruitment in vivo and protects against the development of inflammatory arthritis.

\section{FINAL REMARKS}

Compelling evidence shows that neutrophils are more complex and sophisticated cells than traditionally thought and display phenotypical and functional heterogeneity. The expression level of chemoattractant receptors on neutrophils depends on neutrophil activation and maturation stage and may even follow a circadian rhythm. These receptors and their ligands are not only indispensable for time- and site-dependent neutrophil trafficking but are also critically involved in the regulation of neutrophil activation. Impressive research efforts reporting the effects of genetic ablation or pharmacological inhibition of distinct chemoattractant receptors underscores the idea that, on the one hand, adequate, strictly controlled expression of these
GPCRs is critical to guarantee immune surveillance, while on the other hand, it prevents host damage. It is important to point out that most scientific reports on chemoattractant-induced neutrophil responses in vitro usually consider only one or a limited number of molecules. In vivo, multiple chemoattractants can be released in parallel, affecting release and activity of each other. Members of different chemoattractant families presumably act in concert, though in a potentially sequential and competitive manner, to govern neutrophil trafficking and activation. Locally produced inflammatory mediators and enzymes may modulate the precise biological activities of chemoattractants and their cognate GPCRs. Complement anaphylatoxins, chemokines and chemokine receptors, for instance, are susceptible to posttranslational modifications. Furthermore, the in vivo expression of neutrophil chemoattractants and their receptors is modulated by environmental cues and depends on their location in the body. Indeed, one should discriminate between circulating neutrophils versus tissue-infiltrated cells. Unfortunately, our knowledge regarding the phenotypical and functional features of infiltrated neutrophils is rather limited since most research concerns the study of isolated peripheral blood cells. Although the results from in vivo imaging have greatly improved our understanding of in vivo neutrophil recruitment, one must keep in mind that important differences exist between the murine chemoattractant network and the human equivalent. For example, the most potent human neutrophil-attracting chemokine, i.e., CXCL8, has no functional homolog in mice. Murine and human neutrophils are also different in terms of the number of circulating cells and their morphology. Moreover, it was thought for a long time that, in mice, there was only one receptor homolog for human CXCR1 and CXCR2, and that it bound all ELR-positive CXC chemokines. However, a second murine receptor with high specificity for murine granulocyte chemotactic protein-2 (the functional equivalent of the human CXCL6 that interacts with both CXCR1 and CXCR2 but is often referred to as murine CXCL5) was identified. ${ }^{240}$ However, this receptor has rarely been studied, and it is unknown whether it is a true functional homolog of human CXCR1. A comparable lack of clear homology exists between human and murine ligands for CCR1, CCR2 and CCR5. In humans, two forms of macrophage inflammatory protein-1a exist, i.e., CCL3 and CCL3L1. With regard to receptor specificity, murine CCL3 resembles human CCL3L1 but not human CCL3. ${ }^{241}$ Ultimately, the existence of different neutrophil subsets in vivo, characterized by unique expression patterns of surface molecules, including chemoattractant receptors, would allow the development of targeted therapies preventing uncontrolled inflammation and tissue damage without impairing the immune surveillance of the host.

\section{ACKNOWLEDGEMENTS}

This research was supported by the Research Foundation - Flanders (FWOVlaanderen) (G.0808.18N), the European Union's Horizon 2020 research and innovation program ImmunoAID under grant agreement No 779295 and a "C1" grant (C16/17/010) from KU Leuven. M.M. obtained a PhD fellowship supported by the L'Oréal-UNESCO for Women in Science initiative and the FWO-Vlaanderen. M.G. is a research expert funded by the Rega Foundation.

\section{AUTHOR CONTRIBUTIONS}

M.M. wrote the initial manuscript, which was corrected and modified by M.G. and P.P. All authors approved the final version of the manuscript.

\section{ADDITIONAL INFORMATION}

The online version of this article (https://doi.org/10.1038/s41423-020-0412-0) contains supplementary material.

Competing interests: The authors declare no competing interests. 


\section{REFERENCES}

1. Hidalgo, A., Chilvers, E. R., Summers, C. \& Koenderman, L. The neutrophil life cycle. Trends Immunol. 40, 584-597 (2019).

2. Silvestre-Roig, C., Hidalgo, A. \& Soehnlein, O. Neutrophil heterogeneity: implications for homeostasis and pathogenesis. Blood 127, 2173-2181 (2016).

3. Liew, P. X. \& Kubes, P. The neutrophil's role during health and disease. Physiol. Rev. 99, 1223-1248 (2019).

4. Headland, S. E. \& Norling, L. V. The resolution of inflammation: principles and challenges. Semin. Immunol. 27, 149-160 (2015).

5. Nguyen, G. T., Green, E. R. \& Mecsas, J. Neutrophils to the ROScue: mechanisms of NADPH oxidase activation and bacterial resistance. Front Cell Infect. Microbiol. 7, 373 (2017).

6. Yin, C. \& Heit, B. Armed for destruction: formation, function and trafficking of neutrophil granules. Cell Tissue Res. 371, 455-471 (2018).

7. Ortmann, W. \& Kolaczkowska, E. Age is the work of art? Impact of neutrophil and organism age on neutrophil extracellular trap formation. Cell Tissue Res. 371, 473-488 (2018)

8. Ley, K., Laudanna, C., Cybulsky, M. I. \& Nourshargh, S. Getting to the site of inflammation: the leukocyte adhesion cascade updated. Nat. Rev. Immunol. 7, 678-689 (2007)

9. Filippi, M.-D. Neutrophil transendothelial migration: updates and new perspectives. Blood 133, 2149-2158 (2019).

10. Sadik, C. D. \& Luster, A. D. Lipid-cytokine-chemokine cascades orchestrate leukocyte recruitment in inflammation. J. Leukoc. Biol. 91, 207-215 (2012)

11. Petri, B. \& Sanz, M.-J. Neutrophil chemotaxis. Cell Tissue Res. 371, 425-436 (2018).

12. McDonald, B. et al. Intravascular danger signals guide neutrophils to sites of sterile inflammation. Science 330, 362-366 (2010).

13. Marques, P. E. et al. Chemokines and mitochondrial products activate neutrophils to amplify organ injury during mouse acute liver failure. Hepatology 56, 1971-1982 (2012).

14. Girbl, T. et al. Distinct compartmentalization of the chemokines CXCL1 and CXCL2 and the atypical receptor ACKR1 determine discrete stages of neutrophil diapedesis. Immunity 49, 1062-1076.e6 (2018).

15. Vogt, K. L., Summers, C., Chilvers, E. R. \& Condliffe, A. M. Priming and de-priming of neutrophil responses in vitro and in vivo. Eur. J. Clin. Invest. 48(Suppl 2), e12967 (2018).

16. Subramanian, B. C., Majumdar, R. \& Parent, C. A. The role of the LTB4-BLT1 axis in chemotactic gradient sensing and directed leukocyte migration. Semin. Immunol. 33, 16-29 (2017).

17. Shindo, K., Koide, K. \& Fukumura, M. Enhancement of leukotriene B4 release in stimulated asthmatic neutrophils by platelet activating factor. Thorax $\mathbf{5 2}$, 1024-1029 (1997).

18. Reznichenko, A. \& Korstanje, R. The role of platelet-activating factor in mesangial pathophysiology. Am. J. Pathol. 185, 888-896 (2015).

19. Klos, A., Wende, E., Wareham, K. J. \& Monk, P. N. International union of basic and clinical pharmacology. [corrected]. LXXXVII. Complement peptide C5a, C4a, and C3a receptors. Pharm. Rev. 65, 500-543 (2013).

20. Chen, $K$. et al. Regulation of inflammation by members of the formyl-peptide receptor family. J. Autoimmun. 85, 64-77 (2017).

21. Dahlgren, C., Gabl, M., Holdfeldt, A., Winther, M. \& Forsman, H. Basic characteristics of the neutrophil receptors that recognize formylated peptides, a danger-associated molecular pattern generated by bacteria and mitochondria. Biochem. Pharmacol. 114, 22-39 (2016).

22. Ye, R. D. et al. International union of basic and clinical pharmacology. LXXIII. Nomenclature for the formyl peptide receptor (FPR) family. Pharm. Rev. 61, 119-161 (2009).

23. Hughes, C. E. \& Nibbs, R. J. B. A guide to chemokines and their receptors. FEBS J. 285, 2944-2971 (2018)

24. Russo, R. C., Garcia, C. C., Teixeira, M. M. \& Amaral, F. A. The CXCL8/IL-8 chemokine family and its receptors in inflammatory diseases. Expert Rev. Clin. Immunol. 10, 593-619 (2014).

25. Mortier, A., Van Damme, J. \& Proost, P. Overview of the mechanisms regulating chemokine activity and availability. Immunol. Lett. 145, 2-9 (2012).

26. Janssens, R., Struyf, S. \& Proost, P. The unique structural and functional features of CXCL12. Cell Mol. Immunol. 15, 299-311 (2018).

27. Vanheule, V., Metzemaekers, M., Janssens, R., Struyf, S. \& Proost, P. How posttranslational modifications influence the biological activity of chemokines. Cytokine 109, 29-51 (2018).

28. Brown, S. L. et al. Activation and regulation of platelet-activating factor receptor: role of $\mathrm{G}(\mathrm{i})$ and $\mathrm{G}(\mathrm{q})$ in receptor-mediated chemotactic, cytotoxic, and crossregulatory signals. J. Immunol. 177, 3242-3249 (2006).

29. Futosi, K., Fodor, S. \& Mocsai, A. Reprint of neutrophil cell surface receptors and their intracellular signal transduction pathways. Int. Immunopharmacol. 17, 1185-1197 (2013).
30. Mocsai, A. et al. Differential effects of tyrosine kinase inhibitors and an inhibitor of the mitogen-activated protein kinase cascade on degranulation and superoxide production of human neutrophil granulocytes. Biochem. Pharmacol. 54, 781-789 (1997).

31. Mocsai, A. et al. Kinase pathways in chemoattractant-induced degranulation of neutrophils: the role of p38 mitogen-activated protein kinase activated by Src family kinases. J. Immunol. 164, 4321-4331 (2000).

32. Seifert, R. \& Wenzel-Seifert, K. Unmasking different constitutive activity of four chemoattractant receptors using $\mathrm{Na}+$ as universal stabilizer of the inactive (R) state. Receptors Channels 7, 357-369 (2001)

33. Gurevich, V. V. \& Gurevich, E. V. GPCR signaling regulation: the role of GRKs and arrestins. Front Pharm. 10, 125 (2019).

34. Borroni, E. M., Mantovani, A., Locati, M. \& Bonecchi, R. Chemokine receptors intracellular trafficking. Pharm. Ther. 127, 1-8 (2010).

35. Blackwood, R. A., Hartiala, K. T., Kwoh, E. E., Transue, A. T. \& Brower, R. C. Unidirectional heterologous receptor desensitization between both the fMLP and C5a receptor and the IL-8 receptor. J. Leukoc. Biol. 60, 88-93 (1996).

36. Kitayama, J., Carr, M. W., Roth, S. J., Buccola, J. \& Springer, T. A. Contrasting responses to multiple chemotactic stimuli in transendothelial migration: heterologous desensitization in neutrophils and augmentation of migration in eosinophils. J. Immunol. 158, 2340-2349 (1997).

37. Sogawa, Y., Ohyama, T., Maeda, H. \& Hirahara, K. Inhibition of neutrophil migration in mice by mouse formyl peptide receptors 1 and 2 dual agonist: indication of cross-desensitization in vivo. Immunology 132, 441-450 (2011).

38. Forsman, $\mathrm{H}$. et al. Reactivation of desensitized formyl peptide receptors by platelet activating factor: a novel receptor cross talk mechanism regulating neutrophil superoxide anion production. PLOS ONE 8, e60169 (2013).

39. Holdfeldt, A. et al. Reactivation of Galphai-coupled formyl peptide receptors is inhibited by Galphaq-selective inhibitors when induced by signals generated by the platelet-activating factor receptor. J. Leukoc. Biol. 102, 871-880 (2017).

40. Back, M. et al. Update on leukotriene, lipoxin and oxoeicosanoid receptors: IUPHAR Review 7. Br. J. Pharm. 171, 3551-3574 (2014).

41. El Kebir, D., Gjorstrup, P. \& Filep, J. G. Resolvin E1 promotes phagocytosisinduced neutrophil apoptosis and accelerates resolution of pulmonary inflammation. Proc. Natl. Acad. Sci. USA 109, 14983-14988 (2012).

42. Tarlowe, M. H. et al. Inflammatory chemoreceptor cross-talk suppresses leukotriene B4 receptor 1-mediated neutrophil calcium mobilization and chemotaxis after trauma. J. Immunol. 171, 2066-2073 (2003).

43. Sumida, $\mathrm{H}$. et al. Interplay between CXCR2 and BLT1 facilitates neutrophil infiltration and resultant keratinocyte activation in a murine model of imiquimod-induced psoriasis. J. Immunol. 192, 4361-4369 (2014).

44. Subramanian, B. C., Moissoglu, K. \& Parent, C. A. The LTB4-BLT1 axis regulates the polarized trafficking of chemoattractant GPCRs during neutrophil chemotaxis. J. Cell Sci. 131, jcs217422 (2018).

45. Lv, J. et al. Leukotriene B(4)-leukotriene B(4) receptor axis promotes oxazoloneinduced contact dermatitis by directing skin homing of neutrophils and CD8(+) T cells. Immunology 146, 50-58 (2015).

46. Oyoshi, M. K. et al. Leukotriene B4-driven neutrophil recruitment to the skin is essential for allergic skin inflammation. Immunity 37, 747-758 (2012).

47. Kim, N. D., Chou, R. C., Seung, E., Tager, A. M. \& Luster, A. D. A unique requirement for the leukotriene $\mathrm{B} 4$ receptor $\mathrm{BLT1}$ for neutrophil recruitment in inflammatory arthritis. J. Exp. Med. 203, 829-835 (2006).

48. Deng, B. et al. The leukotriene B4-leukotriene B4 receptor axis promotes cisplatin-induced acute kidney injury by modulating neutrophil recruitment. Kidney Int. 92, 89-100 (2017).

49. Kojo, K. et al. BLT1 signalling protects the liver against acetaminophen hepatotoxicity by preventing excessive accumulation of hepatic neutrophils. Sci. Rep. 6, 29650 (2016).

50. Li, X. et al. Dual role of leukotriene B4 receptor type 1 in experimental sepsis. J. Surg. Res. 193, 902-908 (2015).

51. Saeki, K. \& Yokomizo, T. Identification, signaling, and functions of LTB4 receptors. Semin. Immunol. 33, 30-36 (2017).

52. Melnikova, V. \& Bar-Eli, M. Inflammation and melanoma growth and metastasis: the role of platelet-activating factor (PAF) and its receptor. Cancer Metastasis Rev. 26, 359-371 (2007).

53. Doi, K. et al. Attenuation of folic acid-induced renal inflammatory injury in platelet-activating factor receptor-deficient mice. Am. J. Pathol. 168, 1413-1424 (2006)

54. Ferreira, M. A. N. D. et al. Sponge-induced angiogenesis and inflammation in PAF receptor-deficient mice (PAFR-KO). Br. J. Pharm. 141, 1185-1192 (2004).

55. Souza, D. G. et al. Role of PAF receptors during intestinal ischemia and reperfusion injury. A comparative study between PAF receptor-deficient mice and PAF receptor antagonist treatment. Br. J. Pharm. 139, 733-740 (2003) 
56. Garcia, C. C. et al. Platelet-activating factor receptor plays a role in lung injury and death caused by Influenza A in mice. PLoS Pathog. 6, e1001171 (2010).

57. Guerrero, A. T. et al. The role of PAF/PAFR signaling in zymosan-induced articular inflammatory hyperalgesia. Naunyn Schmiedebergs Arch. Pharmacol. 386, 51-59 (2013).

58. Bachi, A. L. L., Dos Santos, L. C., Nonogaki, S., Jancar, S. \& Jasiulionis, M. G. Apoptotic cells contribute to melanoma progression and this effect is partially mediated by the platelet-activating factor receptor. Mediators Inflamm. 2012, 610371 (2012)

59. Soares, A. C. et al. Role of the platelet-activating factor (PAF) receptor during pulmonary infection with gram negative bacteria. Br. J. Pharm. 137, 621-628 (2002).

60. van Zoelen, M. A. D. et al. Platelet-activating factor receptor contributes to host defense against Pseudomonas aeruginosa pneumonia but is not essential for the accompanying inflammatory and procoagulant response. J. Immunol. 180, 3357-3365 (2008).

61. Guglietta, S. et al. Coagulation induced by C3aR-dependent NETosis drives protumorigenic neutrophils during small intestinal tumorigenesis. Nat. Commun. 7, 11037 (2016).

62. Settmacher, B. et al. Modulation of C3a activity: internalization of the human C3a receptor and its inhibition by C5a. J. Immunol. 162, 7409-7416 (1999).

63. Coulthard, L. G. \& Woodruff, T. M. Is the complement activation product C3a a proinflammatory molecule? Re-evaluating the evidence and the myth. J. Immunol. 194, 3542-3548 (2015).

64. Daffern, P. J., Pfeifer, P. H., Ember, J. A. \& Hugli, T. E. C3a is a chemotaxin for human eosinophils but not for neutrophils. I. C3a stimulation of neutrophils is secondary to eosinophil activation. J. Exp. Med. 181, 2119-2127 (1995).

65. Brennan, F. H. et al. Complement receptor C3aR1 controls neutrophil mobilization following spinal cord injury through physiological antagonism of CXCR2. JCl Insight 4, 98254 (2019).

66. Li, X. X., Lee, J. D., Kemper, C. \& Woodruff, T. M. The complement receptor C5aR2: a powerful modulator of innate and adaptive immunity. J. Immunol. 202, 3339-3348 (2019).

67. Miyabe, Y., Miyabe, C., Mani, V., Mempel, T. R. \& Luster, A. D. Atypical complement receptor $\mathrm{C} 5 \mathrm{aR} 2$ transports $\mathrm{C} 5 \mathrm{a}$ to initiate neutrophil adhesion and inflammation. Sci. Immunol. 4, eaav5951 (2019).

68. Spilberg, I., Mehta, J., Muniain, M. A., Simchowitz, L. \& Atkinson, J. Receptor blockade as a mechanism of deactivation of human neutrophils by pepstatin and formyl-Met-Leu-Phe. Inflammation 8, 73-86 (1984).

69. Binder, R., Kress, A. \& Kirschfink, M. Modulation of C5a-mediated effector functions of human polymorphonuclear leukocytes by tumor necrosis factor alpha and granulocyte macrophage colony-stimulating factor. Exp. Clin. Immunogenet. 16, 212-225 (1999).

70. Guo, R.-F., Riedemann, N. C. \& Ward, P. A. Role of C5a-C5aR interaction in sepsis. Shock 21, 1-7 (2004).

71. Guo, R.-F. et al. Neutrophil C5a receptor and the outcome in a rat model of sepsis. FASEB J. 17, 1889-1891 (2003).

72. Amara, U. et al. Early expression changes of complement regulatory proteins and $C 5 A$ receptor (CD88) on leukocytes after multiple injury in humans. Shock 33, 568-575 (2010).

73. Meddows-Taylor, S., Pendle, S. \& Tiemessen, C. T. Altered expression of CD88 and associated impairment of complement 5a-induced neutrophil responses in human immunodeficiency virus type 1-infected patients with and without pulmonary tuberculosis. J. Infect. Dis. 183, 662-665 (2001).

74. Fortunati, E., Kazemier, K. M., Grutters, J. C., Koenderman, L. \& Van den Bosch van, J. M. M. Human neutrophils switch to an activated phenotype after homing to the lung irrespective of inflammatory disease. Clin. Exp. Immunol. 155, 559-566 (2009).

75. Lewis, S. L., Van Epps, D. E. \& Chenoweth, D. E. C5a receptor modulation on neutrophils and monocytes from chronic hemodialysis and peritoneal dialysis patients. Clin. Nephrol. 26, 37-44 (1986).

76. Seely, A. J. E. et al. Alteration of chemoattractant receptor expression regulates human neutrophil chemotaxis in vivo. Ann. Surg. 235, 550-559 (2002).

77. Monach, P. A. et al. Neutrophils in a mouse model of autoantibody-mediated arthritis: critical producers of Fc receptor gamma, the receptor for $\mathrm{C} 5 \mathrm{a}$, and lymphocyte function-associated antigen 1. Arthritis Rheum. 62, 753-764 (2010).

78. Banda, N. K. et al. Role of C3a receptors, $\mathrm{C} 5$ a receptors, and complement protein C6 deficiency in collagen antibody-induced arthritis in mice. J. Immunol. 188, 1469-1478 (2012)

79. Atkinson, S. M. et al. Treatment with anti-C5aR mAb leads to early-onset clinical and mechanistic effects in the murine delayed-type hypersensitivity arthritis model. Autoimmunity 48, 460-470 (2015).

80. Hornum, L. et al. C5a and C5aR are elevated in joints of rheumatoid and psoriatic arthritis patients, and C5aR blockade attenuates leukocyte migration to synovial fluid. PLOS ONE 12, e0189017 (2017).
81. Arumugam, T. V. et al. Protective effect of a human C5a receptor antagonist against hepatic ischaemia-reperfusion injury in rats. J. Hepatol. 40, 934-941 (2004).

82. Schreiber, A. et al. C5a receptor mediates neutrophil activation and ANCAinduced glomerulonephritis. J. Am. Soc. Nephrol. 20, 289-298 (2009).

83. Bao, L. et al. C5a promotes development of experimental lupus nephritis which can be blocked with a specific receptor antagonist. Eur. J. Immunol. 35, 2496-2506 (2005).

84. Dick, J. et al. C5a receptor 1 promotes autoimmunity, neutrophil dysfunction and injury in experimental anti-myeloperoxidase glomerulonephritis. Kidney Int. 93, 615-625 (2018).

85. Baelder, R. et al. Pharmacological targeting of anaphylatoxin receptors during the effector phase of allergic asthma suppresses airway hyperresponsiveness and airway inflammation. J. Immunol. 174, 783-789 (2005).

86. Staab, E. B., Sanderson, S. D., Wells, S. M. \& Poole, J. A. Treatment with the C5a receptor/CD88 antagonist PMX205 reduces inflammation in a murine model of allergic asthma. Int. Immunopharmacol. 21, 293-300 (2014).

87. Osaka, M. et al. Critical role of the C5a-activated neutrophils in high-fat dietinduced vascular inflammation. Sci. Rep. 6, 21391 (2016).

88. Onnheim, K. et al. A novel receptor cross-talk between the ATP receptor P2Y2 and formyl peptide receptors reactivates desensitized neutrophils to produce superoxide. Exp. Cell Res. 323, 209-217 (2014).

89. Wang, X., Qin, W., Zhang, Y., Zhang, H. \& Sun, B. Endotoxin promotes neutrophil hierarchical chemotaxis via the p38-membrane receptor pathway. Oncotarget 7 , 74247-74258 (2016).

90. Ye, D. et al. Lipocalin-2 mediates non-alcoholic steatohepatitis by promoting neutrophil-macrophage crosstalk via the induction of CXCR2. J. Hepatol. 65, 988-997 (2016).

91. Dai, Y., Major, J., Novotny, M. \& Hamilton, T. A. IL-4 inhibits expression of the formyl peptide receptor gene in mouse peritoneal macrophages. J. Interferon Cytokine Res. 25, 11-19 (2005)

92. Li, S.-Q. et al. The expression of formyl peptide receptor 1 is correlated with tumor invasion of human colorectal cancer. Sci. Rep. 7, 5918 (2017).

93. Loor, F., Tiberghien, F., Wenandy, T., Didier, A. \& Traber, R. Cyclosporins: structure-activity relationships for the inhibition of the human FPR1 formylpeptide receptor. J. Med. Chem. 45, 4613-4628 (2002).

94. Stenfeldt, A.-L. et al. The non-steroidal anti-inflammatory drug piroxicam blocks ligand binding to the formyl peptide receptor but not the formyl peptide receptor like 1. Biochem. Pharmacol. 74, 1050-1056 (2007).

95. Yang, S.-C. et al. Propofol inhibits superoxide production, elastase release, and chemotaxis in formyl peptide-activated human neutrophils by blocking formyl peptide receptor 1. J. Immunol. 190, 6511-6519 (2013).

96. Rajeeve, K., Das, S., Prusty, B. K. \& Rudel, T. Chlamydia trachomatis paralyses neutrophils to evade the host innate immune response. Nat. Microbiol. 3, 824-835 (2018).

97. Kaczmarek, E. et al. A subset of five human mitochondrial formyl peptides mimics bacterial peptides and functionally deactivates human neutrophils. J. Trauma Acute Care Surg. 85, 936-943 (2018).

98. Raoof, M., Zhang, Q., Itagaki, K. \& Hauser, C. J. Mitochondrial peptides are potent immune activators that activate human neutrophils via FPR-1. J. Trauma 68 1324-1328 (2010).

99. Dorward, D. A. et al. Novel role for endogenous mitochondrial formylated peptide-driven formyl peptide receptor 1 signalling in acute respiratory distress syndrome. Thorax 72, 928-936 (2017).

100. Scozzi, D. et al. Mitochondrial damage-associated molecular patterns released by lung transplants are associated with primary graft dysfunction. Am. J. Transplant. 19, 1464-1477 (2019).

101. Zhang, H.-W. et al. RvD1 ameliorates LPS-induced acute lung injury via the suppression of neutrophil infiltration by reducing CXCL2 expression and release from resident alveolar macrophages. Int. Immunopharmacol. 76, 105877 (2019).

102. Bjorkman, L. et al. The proinflammatory activity of recombinant serum amyloid $A$ is not shared by the endogenous protein in the circulation. Arthritis Rheum. 62, 1660-1665 (2010).

103. De Buck, M. et al. $\mathrm{COOH}$-terminal SAA1 peptides fail to induce chemokines but synergize with CXCL8 and CCL3 to recruit leukocytes via FPR2. Blood 131, 439-449 (2018)

104. Walther, A., Riehemann, K. \& Gerke, V. A novel ligand of the formyl peptide receptor: annexin I regulates neutrophil extravasation by interacting with the FPR. Mol. Cell 5, 831-840 (2000)

105. Machado, I. D. et al. Annexin A1 is a physiological modulator of neutrophil maturation and recirculation acting on the CXCR4/CXCL12 Pathway. J. Cell Physiol. 231, 2418-2427 (2016).

106. Liu, M. et al. Formylpeptide receptors are critical for rapid neutrophil mobilization in host defense against Listeria monocytogenes. Sci. Rep. 2, 786 (2012). 
107. Oldekamp, S. et al. Lack of formyl peptide receptor 1 and 2 leads to more severe inflammation and higher mortality in mice with of pneumococcal meningitis. Immunology 143, 447-461 (2014).

108. Lefrancais, E., Mallavia, B., Zhuo, H., Calfee, C. S. \& Looney, M. R. Maladaptive role of neutrophil extracellular traps in pathogen-induced lung injury. JCI Insight $\mathbf{3}$, 98178 (2018)

109. Liu, M. et al. Formylpeptide receptors mediate rapid neutrophil mobilization to accelerate wound healing. PLOS ONE 9, e90613 (2014).

110. Honda, M. et al. Intravital imaging of neutrophil recruitment reveals the efficacy of FPR1 blockade in hepatic ischemia-reperfusion injury. J. Immunol. 198, 1718-1728 (2017).

111. Grommes, J., Drechsler, M. \& Soehnlein, O. CCR5 and FPR1 mediate neutrophil recruitment in endotoxin-induced lung injury. J. Innate Immun. 6, 111-116 (2014).

112. Cardini, S. et al. Genetic ablation of the fpr1 gene confers protection from smoking-induced lung emphysema in mice. Am. J. Respir. Cell Mol. Biol. 47, 332-339 (2012).

113. Di Paola, R. et al. Formyl peptide receptor 1 signalling promotes experimental colitis in mice. Pharmacol. Res 141, 591-601 (2019).

114. Fusco, R. et al. Absence of formyl peptide receptor 1 causes endometriotic lesion regression in a mouse model of surgically-induced endometriosis. Oncotarget 9, 31355-31366 (2018).

115. Wang, X., Qin, W., Song, M., Zhang, Y. \& Sun, B. Exogenous carbon monoxide inhibits neutrophil infiltration in LPS-induced sepsis by interfering with FPR1 via p38 MAPK but not GRK2. Oncotarget 7, 34250-34265 (2016).

116. Bachelerie, F. et al. International Union of Basic and Clinical Pharmacology. [corrected]. LXXXIX. Update on the extended family of chemokine receptors and introducing a new nomenclature for atypical chemokine receptors. Pharm. Rev. 66, 1-79 (2014).

117. Ahuja, S. K. \& Murphy, P. M. The CXC chemokines growth-regulated oncogene (GRO) alpha, GRObeta, GROgamma, neutrophil-activating peptide-2, and epithelial cell-derived neutrophil-activating peptide-78 are potent agonists for the type B, but not the type A, human interleukin-8 receptor. J. Biol. Chem. 271, 20545-20550 (1996).

118. Reyes-Robles, T. et al. Staphylococcus aureus leukotoxin ED targets the chemokine receptors CXCR1 and CXCR2 to kill leukocytes and promote infection. Cell Host Microbe 14, 453-459 (2013).

119. Doroshenko, T. et al. Phagocytosing neutrophils down-regulate the expression of chemokine receptors CXCR1 and CXCR2. Blood 100, 2668-2671 (2002).

120. de Oliveira, S., Rosowski, E. E. \& Huttenlocher, A. Neutrophil migration in infection and wound repair: going forward in reverse. Nat. Rev. Immunol. 16, 378-391 (2016).

121. Khandaker, M. H. et al. Metalloproteinases are involved in lipopolysaccharideand tumor necrosis factor-alpha-mediated regulation of CXCR1 and CXCR2 chemokine receptor expression. Blood 93, 2173-2185 (1999).

122. Bakele, M. et al. An interactive network of elastase, secretases, and PAR-2 protein regulates CXCR1 receptor surface expression on neutrophils. J. Biol. Chem. 289, 20516-20525 (2014).

123. Sabroe, I., Jones, E. C., Whyte, M. K. B. \& Dower, S. K. Regulation of human neutrophil chemokine receptor expression and function by activation of Tolllike receptors 2 and 4. Immunology 115, 90-98 (2005).

124. Schmausser, B. et al. Downregulation of CXCR1 and CXCR2 expression on human neutrophils by Helicobacter pylori: a new pathomechanism in $\mathrm{H}$. pylori infection? Infect. Immun. 72, 6773-6779 (2004).

125. Grutkoski, P. S., Graeber, C. T., D'Amico, R., Keeping, H. \& Simms, H. H. Regulation of IL-8RA (CXCR1) expression in polymorphonuclear leukocytes by hypoxia/ reoxygenation. J. Leukoc. Biol. 65, 171-178 (1999).

126. Stadlbauer, V. et al. Role of Toll-like receptors 2,4 , and 9 in mediating neutrophil dysfunction in alcoholic hepatitis. Am. J. Physiol. Gastrointest. Liver Physiol. 296, G15-G22 (2009).

127. Impellizzieri, D. et al. IL-4 receptor engagement in human neutrophils impairs their migration and extracellular trap formation. J. Allergy Clin. Immunol. 144, 267-279.e4 (2019).

128. Pillay, J. et al. Functional heterogeneity and differential priming of circulating neutrophils in human experimental endotoxemia. J. Leukoc. Biol. 88, 211-220 (2010)

129. Pignatti, P. et al. Downmodulation of CXCL8/LL-8 receptors on neutrophils after recruitment in the airways. J. Allergy Clin. Immunol. 115, 88-94 (2005).

130. Hartl, D. et al. Cleavage of CXCR1 on neutrophils disables bacterial killing in cystic fibrosis lung disease. Nat. Med. 13, 1423-1430 (2007).

131. Godaly, G. et al. Neutrophil recruitment, chemokine receptors, and resistance to mucosal infection. J. Leukoc. Biol. 69, 899-906 (2001).

132. Sherry, B., Dai, W. W., Lesser, M. L. \& Trachtman, H. Dysregulated chemokine receptor expression and chemokine-mediated cell trafficking in pediatric patients with ESRD. Clin. J. Am. Soc. Nephrol. 3, 397-406 (2008).
133. Xu, R. et al. Low expression of CXCR1/2 on neutrophils predicts poor survival in patients with hepatitis B virus-related acute-on-chronic liver failure. Sci. Rep. 6, 38714 (2016).

134. Pereira, R. et al. Neutrophil and monocyte activation in chronic kidney disease patients under hemodialysis and its relationship with resistance to recombinant human erythropoietin and to the hemodialysis procedure. Hemodial. Int. 14, 295-301 (2010).

135. Swamydas, M. et al. CXCR1-mediated neutrophil degranulation and fungal killing promote Candida clearance and host survival. Sci. Transl. Med. 8, 322ra10 (2016).

136. Hauser, C. J. et al. CXCR2 stimulation primes CXCR1 [Ca2+]i responses to IL-8 in human neutrophils. Shock 12, 428-437 (1999).

137. Eash, K. J., Greenbaum, A. M., Gopalan, P. K. \& Link, D. C. CXCR2 and CXCR4 antagonistically regulate neutrophil trafficking from murine bone marrow. J. Clin. Invest. 120, 2423-2431 (2010).

138. Laarman, A. J. et al. Staphylococcus aureus Staphopain A inhibits CXCR2dependent neutrophil activation and chemotaxis. EMBO J. 31, 3607-3619 (2012).

139. Rios-Santos, F. et al. Down-regulation of CXCR2 on neutrophils in severe sepsis is mediated by inducible nitric oxide synthase-derived nitric oxide. Am. J. Respir. Crit. Care Med. 175, 490-497 (2007).

140. Deng, M. et al. Toll-like receptor 4 signaling on dendritic cells suppresses polymorphonuclear leukocyte CXCR2 expression and trafficking via interleukin 10 during intra-abdominal sepsis. J. Infect. Dis. 213, 1280-1288 (2016).

141. Rose, J. J., Foley, J. F., Murphy, P. M. \& Venkatesan, S. On the mechanism and significance of ligand-induced internalization of human neutrophil chemokine receptors CXCR1 and CXCR2. J. Biol. Chem. 279, 24372-24386 (2004).

142. Qiao, H. et al. CXCR2 Expression on neutrophils is upregulated during the relapsing phase of ocular Behcet disease. Curr. Eye Res. 30, 195-203 (2005)

143. Bajrami, B. et al. G-CSF maintains controlled neutrophil mobilization during acute inflammation by negatively regulating CXCR2 signaling. J. Exp. Med. 213, 1999-2018 (2016).

144. Hsieh, S.-C. et al. Abnormal in vitro CXCR2 modulation and defective cationic ion transporter expression on polymorphonuclear neutrophils responsible for hyporesponsiveness to IL-8 stimulation in patients with active systemic lupus erythematosus. Rheumatology 47, 150-157 (2008).

145. Cummings, C. J. et al. Expression and function of the chemokine receptors CXCR1 and CXCR2 in sepsis. J. Immunol. 162, 2341-2346 (1999).

146. Chishti, A. D., Shenton, B. K., Kirby, J. A. \& Baudouin, S. V. Neutrophil chemotaxis and receptor expression in clinical septic shock. Intensive Care Med. 30, 605-611 (2004)

147. Lee, S. K. et al. Phospholipase D2 drives mortality in sepsis by inhibiting neutrophil extracellular trap formation and down-regulating CXCR2. J. Exp. Med. 212, 1381-1390 (2015).

148. Cockx, M. et al. Neutrophils from patients with primary ciliary dyskinesia display reduced chemotaxis to CXCR2 ligands. Front Immunol. 8, 1126 (2017).

149. Sue, R. D. et al. CXCR2 is critical to hyperoxia-induced lung injury. J. Immunol. 172, 3860-3868 (2004).

150. Farooq, S. M. et al. Therapeutic effect of blocking CXCR2 on neutrophil recruitment and dextran sodium sulfate-induced colitis. J. Pharm. Exp. Ther. 329, 123-129 (2009).

151. Ajuebor, M. N., Zagorski, J., Kunkel, S. L., Strieter, R. M. \& Hogaboam, C. M. Contrasting roles for CXCR2 during experimental colitis. Exp. Mol. Pathol. 76, 1-8 (2004).

152. Buanne, $P$. et al. Crucial pathophysiological role of CXCR2 in experimental ulcerative colitis in mice. J. Leukoc. Biol. 82, 1239-1246 (2007).

153. Londhe, V. A. et al. CXCR2 is critical for dsRNA-induced lung injury: relevance to viral lung infection. J. Inflamm. 2, 4 (2005).

154. Belperio, J. A. et al. CXCR2/CXCR2 ligand biology during lung transplant ischemia-reperfusion injury. J. Immunol. 175, 6931-6939 (2005).

155. Belperio, J. A. et al. Role of CXCR2/CXCR2 ligands in vascular remodeling during bronchiolitis obliterans syndrome. J. Clin. Invest. 115, 1150-1162 (2005).

156. Hall, L. R., Diaconu, E., Patel, R. \& Pearlman, E. CXC chemokine receptor 2 but not C-C chemokine receptor 1 expression is essential for neutrophil recruitment to the cornea in helminth-mediated keratitis (river blindness). J. Immunol. 166, 4035-4041 (2001).

157. Nair, P. et al. Safety and efficacy of a CXCR2 antagonist in patients with severe asthma and sputum neutrophils: a randomized, placebo-controlled clinical trial. Clin. Exp. Allergy 42, 1097-1103 (2012).

158. Leaker, B. R., Barnes, P. J. \& O'Connor, B. Inhibition of LPS-induced airway neutrophilic inflammation in healthy volunteers with an oral CXCR2 antagonist. Respir. Res. 14, 137 (2013). 
159. O'Byrne, P. M. et al. Efficacy and safety of a CXCR2 antagonist, AZD5069, in patients with uncontrolled persistent asthma: a randomised, double-blind, placebo-controlled trial. Lancet Respir. Med. 4, 797-806 (2016).

160. Tsai, W. C. et al. CXC chemokine receptor CXCR2 is essential for protective innate host response in murine Pseudomonas aeruginosa pneumonia. Infect. Immun. 68, 4289-4296 (2000).

161. Kielian, T., Barry, B. \& Hickey, W. F. CXC chemokine receptor-2 ligands are required for neutrophil-mediated host defense in experimental brain abscesses. J. Immunol. 166, 4634-4643 (2001).

162. Svensson, M., Irjala, H., Svanborg, C. \& Godaly, G. Effects of epithelial and neutrophil CXCR2 on innate immunity and resistance to kidney infection. Kidney Int. 74, 81-90 (2008).

163. Tateda, K. et al. Chemokine-dependent neutrophil recruitment in a murine model of Legionella pneumonia: potential role of neutrophils as immunoregulatory cells. Infect. Immun. 69, 2017-2024 (2001).

164. Spehlmann, M. E. et al. CXCR2-dependent mucosal neutrophil influx protects against colitis-associated diarrhea caused by an attaching/effacing lesionforming bacterial pathogen. J. Immunol. 183, 3332-3343 (2009).

165. Banerjee, K., Biswas, P. S., Kim, B., Lee, S. \& Rouse, B. T. CXCR2-/- mice show enhanced susceptibility to herpetic stromal keratitis: a role for IL-6-induced neovascularization. J. Immunol. 172, 1237-1245 (2004).

166. Milatovic, S., Nanney, L. B., Yu, Y., White, J. R. \& Richmond, A. Impaired healing of nitrogen mustard wounds in CXCR2 null mice. Wound Repair Regen. 11, 213-219 (2003).

167. Fridlender, Z. G. et al. Polarization of tumor-associated neutrophil phenotype by TGF-beta: 'N1' versus 'N2' TAN. Cancer Cell 16, 183-194 (2009).

168. Raccosta, L. et al. The oxysterol-CXCR2 axis plays a key role in the recruitment of tumor-promoting neutrophils. J. Exp. Med. 210, 1711-1728 (2013).

169. Gong, L. et al. Promoting effect of neutrophils on lung tumorigenesis is mediated by CXCR2 and neutrophil elastase. Mol. Cancer 12, 154 (2013).

170. Tazzyman, S. et al. Inhibition of neutrophil infiltration into A549 lung tumors in vitro and in vivo using a CXCR2-specific antagonist is associated with reduced tumor growth. Int. J. Cancer 129, 847-858 (2011).

171. Jamieson, T. et al. Inhibition of CXCR2 profoundly suppresses inflammation driven and spontaneous tumorigenesis. J. Clin. Invest. 122, 3127-3144 (2012).

172. Steele, C. W. et al. CXCR2 inhibition profoundly suppresses metastases and augments immunotherapy in pancreatic ductal Adenocarcinoma. Cancer Cell 29, 832-845 (2016).

173. Yu, P. F. et al. TNFalpha-activated mesenchymal stromal cells promote breast cancer metastasis by recruiting CXCR2(+) neutrophils. Oncogene 36, $482-490$ (2017).

174. Li, L. et al. CXCR2-CXCL1 axis is correlated with neutrophil infiltration and predicts a poor prognosis in hepatocellular carcinoma. J. Exp. Clin. Cancer Res. 34, 129 (2015).

175. Metzemaekers, M., Vanheule, V., Janssens, R., Struyf, S. \& Proost, P. Overview of the mechanisms that may contribute to the non-redundant activities of interferon-inducible CXC chemokine receptor 3 ligands. Front Immunol. 8, 1970 (2017).

176. Hartl, D. et al. Infiltrated neutrophils acquire novel chemokine receptor expression and chemokine responsiveness in chronic inflammatory lung diseases. J. Immunol. 181, 8053-8067 (2008).

177. Ichikawa, A. et al. CXCL10-CXCR3 enhances the development of neutrophilmediated fulminant lung injury of viral and nonviral origin. Am. J. Respir. Crit. Care Med. 187, 65-77 (2013).

178. Rudd, J. M. et al. Neutrophils induce a novel chemokine receptors repertoire during influenza pneumonia. Front Cell Infect. Microbiol. 9, 108 (2019).

179. Chami, B. et al. CXCR3 plays a critical role for host protection against Salmonellosis. Sci. Rep. 7, 10181 (2017).

180. Forster, R. et al. Intracellular and surface expression of the HIV-1 coreceptor CXCR4/fusin on various leukocyte subsets: rapid internalization and recycling upon activation. J. Immunol. 160, 1522-1531 (1998).

181. Gouwy, M., Struyf, S., Catusse, J., Proost, P. \& Van Damme, J. Synergy between proinflammatory ligands of $\mathrm{G}$ protein-coupled receptors in neutrophil activation and migration. J. Leukoc. Biol. 76, 185-194 (2004)

182. Bruhl, $\mathrm{H}$. et al. Post-translational and cell type-specific regulation of CXCR4 expression by cytokines. Eur. J. Immunol. 33, 3028-3037 (2003).

183. Nagase, $\mathrm{H}$. et al. Cytokine-mediated regulation of CXCR4 expression in human neutrophils. J. Leukoc. Biol. 71, 711-717 (2002).

184. Machado, I. D. et al. Alterations in the profile of blood neutrophil membrane receptors caused by in vivo adrenocorticotrophic hormone actions. Am. J. Physiol. Endocrinol. Metab. 307, E754-E763 (2014).

185. Suratt, B. T. et al. Role of the CXCR4/SDF-1 chemokine axis in circulating neutrophil homeostasis. Blood 104, 565-571 (2004).

186. Wetzler, M. et al. A new familial immunodeficiency disorder characterized by severe neutropenia, a defective marrow release mechanism, and hypogammaglobulinemia. Am. J. Med. 89, 663-672 (1990).
187. Martin, C. et al. Chemokines acting via CXCR2 and CXCR4 control the release of neutrophils from the bone marrow and their return following senescence. Immunity 19, 583-593 (2003).

188. Weisel, K. C. et al. Modulation of CXC chemokine receptor expression and function in human neutrophils during aging in vitro suggests a role in their clearance from circulation. Mediators Inflamm. 2009, 790174 (2009).

189. Zhang, D. et al. Neutrophil ageing is regulated by the microbiome. Nature $\mathbf{5 2 5}$, 528-532 (2015).

190. Furze, R. C. \& Rankin, S. M. The role of the bone marrow in neutrophil clearance under homeostatic conditions in the mouse. FASEB J. 22, 3111-3119 (2008).

191. Kim, H. K., De La Luz Sierra, M., Williams, C. K., Gulino, A. V. \& Tosato, G. G-CSF down-regulation of CXCR4 expression identified as a mechanism for mobilization of myeloid cells. Blood 108, 812-820 (2006).

192. Lum, J. J., Bren, G., McClure, R. \& Badley, A. D. Elimination of senescent neutrophils by TNF-related apoptosis-inducing [corrected] ligand. J. Immunol. 175, 1232-1238 (2005).

193. Wang, J. et al. Visualizing the function and fate of neutrophils in sterile injury and repair. Science 358, 111-116 (2017).

194. Kim, J. H. et al. Aged polymorphonuclear leukocytes cause fibrotic interstitial lung disease in the absence of regulation by B cells. Nat. Immunol. 19, 192-201 (2018).

195. Eash, K. J., Means, J. M., White, D. W. \& Link, D. C. CXCR4 is a key regulator of neutrophil release from the bone marrow under basal and stress granulopoiesis conditions. Blood 113, 4711-4719 (2009).

196. Yamada, M. et al. The increase in surface CXCR4 expression on lung extravascular neutrophils and its effects on neutrophils during endotoxin-induced lung injury. Cell Mol. Immunol. 8, 305-314 (2011).

197. Petty, J. M. et al. Pulmonary stromal-derived factor-1 expression and effect on neutrophil recruitment during acute lung injury. J. Immunol. 178, 8148-8157 (2007).

198. Radermecker C, et al. Locally instructed CXCR4(hi) neutrophils trigger environment-driven allergic asthma through the release of neutrophil extracellular traps. Nat. Immunol. 20, 1444-1455 (2019).

199. Lenoir, M., Djerdjouri, B. \& Perianin, A. Stroma cell-derived factor 1alpha mediates desensitization of human neutrophil respiratory burst in synovial fluid from rheumatoid arthritic patients. J. Immunol. 172, 7136-7143 (2004).

200. McDermott, D. H. et al. Severe congenital neutropenia resulting from G6PC3 deficiency with increased neutrophil CXCR4 expression and myelokathexis. Blood 116, 2793-2802 (2010).

201. Wang, A. et al. CXCR4/CXCL12 hyperexpression plays a pivotal role in the pathogenesis of lupus. J. Immunol. 182, 4448-4458 (2009).

202. Delano, M. J. et al. Neutrophil mobilization from the bone marrow during polymicrobial sepsis is dependent on CXCL12 signaling. J. Immunol. 187, 911-918 (2011).

203. Weisenburger-Lile, D. et al. Harmful neutrophil subsets in patients with ischemic stroke: association with disease severity. Neurol. Neuroimmunol. Neuroinflamm. 6, e571 (2019).

204. Massena, S. et al. Identification and characterization of VEGF-A-responsive neutrophils expressing CD49d, VEGFR1, and CXCR4 in mice and humans. Blood 126, 2016-2026 (2015).

205. Zhang, S., Youn, B. S., Gao, J. L., Murphy, P. M. \& Kwon, B. S. Differential effects of leukotactin-1 and macrophage inflammatory protein-1 alpha on neutrophils mediated by CCR1. J. Immunol. 162, 4938-4942 (1999).

206. Lee, S. C. et al. Cutaneous injection of human subjects with macrophage inflammatory protein-1 alpha induces significant recruitment of neutrophils and monocytes. J. Immunol. 164, 3392-3401 (2000)

207. Jose, R. et al. Regulation of neutrophilic inflammation in lung injury induced by community-acquired pneumonia. Lancet 385 (Suppl), S52 (2015).

208. Yamamoto, T. et al. Loss of SMAD4 promotes lung metastasis of colorectal cancer by accumulation of CCR1+ tumor-associated neutrophils through CCL15-CCR1 axis. Clin. Cancer Res. 23, 833-844 (2017).

209. Reichel, C. A. et al. Chemokine receptors C cr1, Ccr2, and Ccr5 mediate neutrophil migration to postischemic tissue. J. Leukoc. Biol. 79, 114-122 (2006).

210. Reichel, C. A. et al. C-C motif chemokine CCL3 and canonical neutrophil attractants promote neutrophil extravasation through common and distinct mechanisms. Blood 120, 880-890 (2012).

211. Ramos, M. V. et al. Chemokine receptor CCR1 disruption limits renal damage in a murine model of hemolytic uremic syndrome. Am. J. Pathol. 180, 1040-1048 (2012).

212. Kaesler, S. et al. The chemokine receptor CCR1 is strongly up-regulated after skin injury but dispensable for wound healing. Wound Repair Regen. 12, 193-204 (2004).

213. Johnston, B. et al. Chronic inflammation upregulates chemokine receptors and induces neutrophil migration to monocyte chemoattractant protein-1. J. Clin. Invest. 103, 1269-1276 (1999).

214. $\mathrm{Xu}, \mathrm{P}$. et al. CCR2 dependent neutrophil activation and mobilization rely on TLR4-p38 axis during liver ischemia-reperfusion injury. Am. J. Transl. Res. 9, 2878-2890 (2017). 
Neutrophil chemoattractant receptors in health and disease: double-edged... M Metzemaekers et al.

450

215. Dimitrijevic, O. B., Stamatovic, S. M., Keep, R. F. \& Andjelkovic, A. V. Absence of the chemokine receptor CCR2 protects against cerebral ischemia/reperfusion injury in mice. Stroke 38, 1345-1353 (2007).

216. Speyer, C. L. et al. Novel chemokine responsiveness and mobilization of neutrophils during sepsis. Am. J. Pathol. 165, 2187-2196 (2004).

217. Feterowski, C. et al. CC chemokine receptor 2 regulates leukocyte recruitment and IL-10 production during acute polymicrobial sepsis. Eur. J. Immunol. 34, 3664-3673 (2004).

218. Souto, F. O. et al. Essential role of CCR2 in neutrophil tissue infiltration and multiple organ dysfunction in sepsis. Am. J. Respir. Crit. Care Med. 83, 234-242 (2011).

219. Talbot, J. et al. CCR2 expression in neutrophils plays a critical role in their migration into the joints in rheumatoid arthritis. Arthritis Rheumatol. 67, 1751-1759 (2015).

220. Struyf, S. et al. Diverging binding capacities of natural LD78beta isoforms of macrophage inflammatory protein-1alpha to the CC chemokine receptors 1, 3 and 5 affect their anti-HIV-1 activity and chemotactic potencies for neutrophils and eosinophils. Eur. J. Immunol. 31, 2170-2178 (2001).

221. Loetscher, P. et al. The ligands of CXC chemokine receptor 3, I-TAC, Mig, and IP10, are natural antagonists for CCR3. J. Biol. Chem. 276, 2986-2991 (2001).

222. Bonecchi, R. et al. Up-regulation of CCR1 and CCR3 and induction of chemotaxis to CC chemokines by IFN-gamma in human neutrophils. J. Immunol. 162, 474-479 (1999).

223. Menzies-Gow, A. et al. Eotaxin (CCL11) and eotaxin-2 (CCL24) induce recruitment of eosinophils, basophils, neutrophils, and macrophages as well as features of early- and late-phase allergic reactions following cutaneous injection in human atopic and nonatopic volunteers. J. Immunol. 169, 2712-2718 (2002).

224. Alonzo, F. III et al. CCR5 is a receptor for Staphylococcus aureus leukotoxin ED. Nature 493, 51-55 (2013).

225. Ottonello, L. et al. CCL3 (MIP-1alpha) induces in vitro migration of GM-CSFprimed human neutrophils via CCR5-dependent activation of ERK 1/2. Cell Signal. 17, 355-363 (2005).

226. Ariel, A. et al. Apoptotic neutrophils and T cells sequester chemokines during immune response resolution through modulation of CCR5 expression. Nat. Immunol. 7, 1209-1216 (2006).
227. Auer, J. et al. Expression and regulation of CCL18 in synovial fluid neutrophils of patients with rheumatoid arthritis. Arthritis Res. Ther. 9, R94 (2007).

228. Eruslanov, E. B. et al. Tumor-associated neutrophils stimulate T cell responses in early-stage human lung cancer. J. Clin. Invest. 124, 5466-5480 (2014).

229. Bonecchi, R. \& Graham, G. J. Atypical chemokine receptors and their roles in the resolution of the inflammatory response. Front Immunol. 7, 224 (2016).

230. Nibbs, R. J. B. \& Graham, G. J. Immune regulation by atypical chemokine receptors. Nat. Rev. Immunol. 13, 815-829 (2013).

231. Bachelerie, F. et al. An atypical addition to the chemokine receptor nomenclature: IUPHAR Review 15. Br. J. Pharm. 172, 3945-3949 (2015).

232. Luo, H., Chaudhuri, A., Zbrzezna, V., He, Y. \& Pogo, A. O. Deletion of the murine Duffy gene (Dfy) reveals that the Duffy receptor is functionally redundant. Mol. Cell Biol. 20, 3097-3101 (2000).

233. Mei, J. et al. CXCL5 regulates chemokine scavenging and pulmonary host defense to bacterial infection. Immunity 33, 106-117 (2010).

234. Lee, K. M., Nibbs, R. J. B. \& Graham, G. J. D6: the 'crowd controller' at the immune gateway. Trends Immunol. 34, 7-12 (2013).

235. Rot, A. et al. Cell-autonomous regulation of neutrophil migration by the D6 chemokine decoy receptor. J. Immunol. 190, 6450-6456 (2013).

236. Castanheira, F. V. E. S. et al. The atypical chemokine receptor ACKR2 is protective against sepsis. Shock 49, 682-689 (2018).

237. Massara, M. et al. ACKR2 in hematopoietic precursors as a checkpoint of neutrophil release and anti-metastatic activity. Nat. Commun. 9, 676 (2018).

238. Del Prete, A., Bonecchi, R., Vecchi, A., Mantovani, A. \& Sozzani, S. CCRL2, a fringe member of the atypical chemoattractant receptor family. Eur. J. Immunol. 43, 1418-1422 (2013).

239. Del Prete, A. et al. The atypical receptor CCRL2 is required for CXCR2-dependent neutrophil recruitment and tissue damage. Blood 130, 1223-1234 (2017).

240. Fan, X. et al. Murine CXCR1 is a functional receptor for GCP-2/CXCL6 and interleukin-8/CXCL8. J. Biol. Chem. 282, 11658-11666 (2007).

241. Menten, P. et al. The LD78beta isoform of MIP-1alpha is the most potent CCR5 agonist and HIV-1-inhibiting chemokine. J. Clin. Invest. 104, R1-R5 (1999). 